\title{
PARAMETER ESTIMATION FOR STOCHASTIC WAVE EQUATION BASED ON OBSERVATION WINDOW
}

\author{
JOSEF JANÁK
}

\begin{abstract}
Statistical inference for a linear stochastic hyperbolic equation with two unknown parameters is studied. Based on observation of coordinates of the solution or their linear combination, minimum contrast estimators are introduced. Strong consistency and asymptotic normality is proved. The results are applied to stochastic wave equation perturbed by Brownian noise and they are illustrated by a numerical simulation.
\end{abstract}

\section{INTRODUCTION}

Statistical inference for stochastic partial differential equations driven by standard Brownian motion has been recently extensively studied. This paper presents results, which are interesting mostly for two reasons. In the first place, many authors use maximum likelihood estimator (MLE) for the estimation of unknown parameters in stochastic partial differential equations (SPDEs) (for example [12]), however we are interested in minimum contrast estimator (MCE). This type of estimator has been studied since 1980's (see the "pioneering" papers [5] and [6]), but there are also more recent works. For example in [11] and [10, the (MCE) is studied even for the SPDEs driven by fractional Brownian motion.

Secondly, many authors concentrate on stochastic parabolic equations (see [2]), but stochastic hyperbolic equations were not paid too much attention to. We may mention [8] or [9], but, again, only the (MLE) is investigated. Therefore the topic of (MCE) for stochastic hyperbolic equations (such as wave equation or plate equation) is rather new, even if the driving process is "only" standard Brownian motion.

In this work, we study parameter estimation for SPDEs of second order in time, in particular, for the following wave equation with strong damping

$$
\begin{aligned}
\frac{\partial^{2} u}{\partial t^{2}}(t, \xi) & =b \Delta u(t, \xi)-2 a \frac{\partial u}{\partial t}(t, \xi)+\eta(t, \xi), \quad(t, \xi) \in \mathbb{R}_{+} \times D \\
u(0, \xi) & =u_{1}(\xi), \quad \xi \in D \\
\frac{\partial u}{\partial t}(0, \xi) & =u_{2}(\xi), \quad \xi \in D \\
u(t, \xi) & =0, \quad(t, \xi) \in \mathbb{R}_{+} \times \partial D
\end{aligned}
$$

Date: June 21, 2018.

2000 Mathematics Subject Classification. 62M05, 93E10, 60G35, 60H15.

Key words and phrases. Parameter estimation, strong consistency, asymptotic normality.

This paper has been produced with contribution of long term institutional support of research activities by Faculty of Informatics and Statistics, University of Economics, Prague.

This paper was supported by the GAČR Grant no. 15-08819S. 
where $D \subset \mathbb{R}^{d}$ is a bounded domain with a smooth boundary and $\eta$ is a random noise.

The aim of the paper is to provide strongly consistent estimators of unknown parameters $a$ and $b$, based on the observation of the trajectory of the solution to (1.1) up to time $T$. Nevertheless, unlike our earlier work [4, where the estimators were dependent on the observation of the norm of the solution, the present estimators will depend only on knowledge of some modes of the solution or their linear combinations (i.e., "observation window"). In order to do so, we follow up the work 4], where the form of strongly continuous semigroup $(S(t), t \geqslant 0)$ generated by the operator in the drift part was found and the form of the covariance operator $Q_{\infty}^{(a, b)}$, the covariance operator of the invariant measure of the system (1.1), was computed. Then we will use the ergodicity of the solution and an appropriate ergodic theorem.

The paper is organized as follows. The Section 2 summarizes some basic facts on stochastic linear partial differential equations (which is mostly due to [1]) as well as setup and assumptions on the model, together with the form of the covariance operator $Q_{\infty}^{(a, b)}$ (which is acquired from [4). In Section 3, the family of strongly consistent estimators $\left(\bar{a}_{T}, \bar{b}_{T}\right)$ is derived. It is a specification of the general result from [10] to the present (hyperbolic) case. Moreover, using the "observation windows" of some special forms, even more estimators may be obtained and we present them with certain estimation strategies.

The asymptotic normality of the proposed estimators is proved in Section 4 In the end of this section, we show how these general results may be simplified for even more concrete "observation coordinates". In Section 5 we consider the basic example of wave equation, where our general results may be applied. These results are illustrated by some numerical simulations in Section 6 .

Let us also introduce some notation. If $U$ and $V$ are Hilbert spaces then $\mathcal{L}(U, V)$, $\mathcal{L}_{2}(U, V)$ and $\mathcal{L}_{1}(U, V)$ denote the respective spaces of all linear bounded, HilbertSchmidt and trace class operators mapping $U$ to $V$. Also, $\mathcal{L}(V)$ stands for $\mathcal{L}(V, V)$, etc.

\section{Preliminaries}

Given separable Hilbert spaces $U$ and $V$, we consider the equation

$$
\begin{aligned}
d X(t) & =\mathcal{A} X(t) d t+\Phi d B(t), \\
X(0) & =x_{0},
\end{aligned}
$$

where $(B(t), t \geqslant 0)$ is a standard cylindrical Brownian motion on $U, \mathcal{A}: \operatorname{Dom}(\mathcal{A}) \rightarrow$ $V, \operatorname{Dom}(\mathcal{A}) \subset V, \mathcal{A}$ is the infinitesimal generator of a strongly continuous semigroup $(S(t), t \geqslant 0)$ on $V, \Phi \in \mathcal{L}(U, V)$ and $x_{0} \in V$ is a random variable. We assume that $\mathbb{E}\left\|x_{0}\right\|_{V}^{2}<\infty$ and that $x_{0}$ and $(B(t), t \geqslant 0)$ are stochastically independent.

We impose the following two conditions:

(A1) $\Phi \in \mathcal{L}_{2}(U, V)$

(A2) There exist constants $K>0$ and $\rho>0$ such that

$$
\|S(t)\|_{\mathcal{L}(V)} \leqslant K e^{-\rho t}
$$

holds for all $t \geqslant 0$.

The condition (A1) means that the perturbing noise is, in fact, a genuine $V$ valued Brownian motion and the condition (A2) is the exponential stability of the semigroup generated by $\mathcal{A}$. 
The following two Propositions describe the form of a mild solution to the equation (2.1) and its invariant measure (cf. [1]).

Proposition 2.1. If (A1) is satisfied then equation (2.1) admits a mild solution

$$
X^{x_{0}}(t)=S(t) x_{0}+Z(t), \quad t \geqslant 0,
$$

where $(Z(t), t \geqslant 0)$ is the convolution integral

$$
Z(t)=\int_{0}^{t} S(t-u) \Phi d B(u), \quad t \geqslant 0 .
$$

The process $(Z(t), t \geqslant 0)$ is a $V$-continuous centered Gaussian process with covariance operator given by the formula

$$
Q_{t}=\int_{0}^{t} S(u) \Phi \Phi^{*} S^{*}(u) d u
$$

Proposition 2.2. If (A1), (A2) are satisfied then there is a unique invariant measure $\mu_{\infty}=N\left(0, Q_{\infty}\right)$ for the equation (2.1) and

$$
w^{*}-\lim _{t \rightarrow \infty} \mu_{t}^{x_{0}}=\mu_{\infty}
$$

for each initial condition $x_{0} \in V$, where $\mu_{t}^{x_{0}}=\operatorname{Law}\left(X^{x_{0}}(t)\right)$ and Law $(\cdot)$ denotes the probability distribution.

The covariance operator $Q_{\infty}$ takes the form

$$
Q_{\infty}=\int_{0}^{\infty} S(t) \Phi \Phi^{*} S^{*}(t) d t
$$

To interpret stochastic wave equation (1.1) rigorously, we rewrite it as a first order system in a standard way. Assume that $\left\{e_{n}, n \in \mathbb{N}\right\}$ is an orthonormal basis in $L^{2}(D)$ and the operator $A: \operatorname{Dom}(A) \subset L^{2}(D) \rightarrow L^{2}(D)$ is such that

(A3) $A e_{n}=-\alpha_{n} e_{n}, \quad \forall n \in \mathbb{N} \quad \alpha_{n}>0, \quad \alpha_{n} \rightarrow \infty$ for $n \rightarrow \infty$.

These assumptions cover the case when the set $D \subset \mathbb{R}^{d}$ is open, bounded and the boundary $\partial D$ is sufficiently smooth, the operator $A=\left.\Delta\right|_{\operatorname{Dom}(A)}$ and $\operatorname{Dom}(A)=$ $H^{2}(D) \cap H_{0}^{1}(D)$.

Next let us assume that $\Phi_{1}$ is a Hilbert-Schmidt operator on $L^{2}(D)$ such that $Q=\Phi_{1} \Phi_{1}^{*}$ satisfies

(A4) $Q e_{n}=\lambda_{n} e_{n}, \quad \forall n \in \mathbb{N} \quad \lambda_{n}>0, \quad \sum_{n=1}^{\infty} \lambda_{n}<\infty$.

The assumption (A4) means that we consider so-called "diagonal case", i.e., operators $A$ and $Q$ have common set of eigenvectors $\left\{e_{n}, n \in \mathbb{N}\right\}$.

Consider the Hilbert space $V=\operatorname{Dom}\left((-A)^{\frac{1}{2}}\right) \times L^{2}(D)$ endowed with the inner product

$$
\begin{aligned}
\left\langle\left(\begin{array}{l}
x_{1} \\
x_{2}
\end{array}\right),\left(\begin{array}{l}
y_{1} \\
y_{2}
\end{array}\right)\right\rangle_{V} & =\left\langle x_{1}, y_{1}\right\rangle_{\operatorname{Dom}\left((-A)^{\frac{1}{2}}\right)}+\left\langle x_{2}, y_{2}\right\rangle_{L^{2}(D)} \\
& =\left\langle(-A)^{\frac{1}{2}} x_{1},(-A)^{\frac{1}{2}} y_{1}\right\rangle_{L^{2}(D)}+\left\langle x_{2}, y_{2}\right\rangle_{L^{2}(D)}
\end{aligned}
$$

for any $\left(x_{1}, x_{2}\right)^{\top},\left(y_{1}, y_{2}\right)^{\top} \in V$. 
Also, consider the linear equation

$$
\begin{gathered}
d X(t)=\mathcal{A} X(t) d t+\Phi d B(t), \\
X(0)=x_{0}=\left(\begin{array}{l}
u_{1} \\
u_{2}
\end{array}\right),
\end{gathered}
$$

where the linear operator $\mathcal{A}: \operatorname{Dom}(\mathcal{A})=\operatorname{Dom}(A) \times \operatorname{Dom}\left((-A)^{\frac{1}{2}}\right) \rightarrow V$ is defined by

$$
\mathcal{A} x=\mathcal{A}\left(\begin{array}{l}
x_{1} \\
x_{2}
\end{array}\right)=\left(\begin{array}{cc}
0 & I \\
b A & -2 a I
\end{array}\right)\left(\begin{array}{l}
x_{1} \\
x_{2}
\end{array}\right), \quad \forall x=\left(\begin{array}{l}
x_{1} \\
x_{2}
\end{array}\right) \in \operatorname{Dom}(\mathcal{A}),
$$

$a>0, b>0$ are unknown parameters (which are to be estimated), $u_{1} \in \operatorname{Dom}\left((-A)^{\frac{1}{2}}\right)$, $u_{2} \in L^{2}(D), x_{0}=\left(u_{1}, u_{2}\right)^{\top} \in V$ satisfies $\mathbb{E}\left\|x_{0}\right\|_{V}^{2}<\infty$, where $\|\cdot\|_{V}:=\sqrt{\langle\cdot, \cdot\rangle_{V}}$, and the linear operator $\Phi: U=V \rightarrow V$ is defined by

$$
\Phi=\left(\begin{array}{cc}
0 & 0 \\
0 & \Phi_{1}
\end{array}\right) .
$$

With no loss of generality, we assume that the driving process in (2.2) takes the form $(0, B(t))^{\top}$, where $(B(t), t \geqslant 0)$ is a standard cylindrical Brownian motion on $L^{2}(D)$.

Note that since the operator $\Phi_{1}$ is Hilbert-Schmidt in $L^{2}(D)$, the operator $\Phi$ is Hilbert-Schmidt in $V$. Also note that the orthonormal basis of the space $\operatorname{Dom}\left((-A)^{\frac{1}{2}}\right)$ is $\left\{f_{n}, n \in \mathbb{N}\right\}$, where $f_{n}=\frac{1}{\sqrt{\alpha_{n}}} e_{n}$.

The operator $\mathcal{A}$ is the infinitesimal generator of the strongly continuous semigroup $(S(t), t \geqslant 0)$ on $V$, which is also exponentially stable (see [4, Theorem 3.10). Its exact form is not needed in the sequel, however we will need the formula for the covariance operator $Q_{\infty}^{(a, b)}$.

Theorem 2.3. If (A1) - (A4) are satisfied then there is a unique invariant measure $\mu_{\infty}^{(a, b)}=N\left(0, Q_{\infty}^{(a, b)}\right)$ for the equation (2.2) and

$$
w^{*}-\lim _{t \rightarrow \infty} \mu_{t}^{x_{0}}=\mu_{\infty}^{(a, b)}
$$

for each initial condition $x_{0} \in V$. The covariance operator $Q_{\infty}^{(a, b)}$ takes the form

$Q_{\infty}^{(a, b)}\left(\begin{array}{c}x_{1} \\ x_{2}\end{array}\right)=\sum_{n=1}^{\infty} \sum_{k=1}^{\infty} \frac{\left\langle Q e_{n}, e_{k}\right\rangle_{L^{2}(D)}}{b^{2}\left(\alpha_{n}-\alpha_{k}\right)^{2}+8 a^{2} b\left(\alpha_{n}+\alpha_{k}\right)} \times$

$$
\left(\begin{array}{c}
4 a \alpha_{n}\left\langle x_{1}, e_{n}\right\rangle_{L^{2}(D)} e_{k}+b\left(\alpha_{k}-\alpha_{n}\right)\left\langle x_{2}, e_{n}\right\rangle_{L^{2}(D)} e_{k} \\
b \alpha_{n}\left(\alpha_{n}-\alpha_{k}\right)\left\langle x_{1}, e_{n}\right\rangle_{L^{2}(D)} e_{k}+2 a b\left(\alpha_{n}+\alpha_{k}\right)\left\langle x_{2}, e_{n}\right\rangle_{L^{2}(D)} e_{k}
\end{array}\right),
$$

for any $\left(x_{1}, x_{2}\right)^{\top} \in V$.

Proof. See [4, Theorem 3.11.

Since we consider only the diagonal case, we will be working with the simplified version of the above formula, that is

$$
Q_{\infty}^{(a, b)}=\left(\begin{array}{cc}
\frac{1}{4 a b} Q & 0 \\
0 & \frac{1}{4 a} Q
\end{array}\right)
$$




\section{Parameter estimation}

Consider the stochastic differential equation (2.2) with the parameters $a>0$, $b>0$ unknown. Our aim is to propose strongly consistent estimators of these parameters based on observation of the trajectory through some "observation window" $z \in V$.

More specifically, let $0 \neq z \in V$ be arbitrary and consider that we are able to track the trajectory of the process $\left(\left\langle X^{x_{0}}(t), z\right\rangle_{V}, 0 \leqslant t \leqslant T\right)$ up to time $T$.

Since the linear differential equation (2.2) has unique invariant measure $\mu_{\infty}^{(a, b)}$, we may use the following ergodic theorem for arbitrary solution (see [10, Theorem 4.9).

Theorem 3.1. Let $\left(X^{x_{0}}(t), t \geqslant 0\right)$ be a solution to (2.2) with $\Phi \in \mathcal{L}_{2}(U, V)$. Let $\varrho: V \rightarrow \mathbb{R}$ be a functional satisfying the following local Lipschitz condition: let there exist real constants $K>0$ and $m \geqslant 0$ such that

$$
|\varrho(x)-\varrho(y)| \leqslant K\|x-y\|_{V}\left(1+\|x\|_{V}^{m}+\|y\|_{V}^{m}\right)
$$

for all $x, y \in V$. Then

$$
\lim _{T \rightarrow \infty} \frac{1}{T} \int_{0}^{T} \varrho\left(X^{x_{0}}(t)\right) d t=\int_{V} \varrho(y) \mu_{\infty}(d y), \quad \mathbb{P}-a . s .
$$

for all $x_{0} \in V$.

Let $z \in V$ be arbitrary. Using a functional $\varrho: V \rightarrow \mathbb{R}, \varrho(y)=\langle y, z\rangle_{V}^{2}, y \in V$, all the conditions of Theorem 3.1 will be satisfied with $m=1$ and

$$
\begin{aligned}
\lim _{T \rightarrow \infty} \frac{1}{T} \int_{0}^{T} \varrho\left(X^{x_{0}}(t)\right) d t & =\lim _{T \rightarrow \infty} \frac{1}{T} \int_{0}^{T}\left\langle X^{x_{0}}(t), z\right\rangle_{V}^{2} d t \\
& =\int_{V}\langle y, z\rangle_{V}^{2} \mu_{\infty}^{(a, b)}(d y) \\
& =\left\langle Q_{\infty}^{(a, b)} z, z\right\rangle_{V}, \quad \mathbb{P}-\text { a.s. }
\end{aligned}
$$

Based on above convergence, some strongly consistent estimators of parameters $a$ and $b$ may be proposed.

Theorem 3.2. Let $z \in V$ be arbitrary, $z=\left(z_{1}, z_{2}\right)^{\top}$, where $z_{1} \in \operatorname{Dom}\left((-A)^{\frac{1}{2}}\right)$, $z_{2} \in L^{2}(D)$. Define

$$
J_{T}=\frac{1}{T} \int_{0}^{T}\left\langle X^{x_{0}}(t), z\right\rangle_{V}^{2} d t
$$

1) If $z \neq 0$ then the process

$$
\bar{a}_{T}=\frac{1}{4 b J_{T}}\left\langle Q z_{1}, z_{1}\right\rangle_{\operatorname{Dom}\left((-A)^{\frac{1}{2}}\right)}+\frac{1}{4 J_{T}}\left\langle Q z_{2}, z_{2}\right\rangle_{L^{2}(D)}
$$

is strongly consistent estimator of the parameter a, i.e., $\bar{a}_{T} \rightarrow$ a, $\mathbb{P}$-a.s. as $T \rightarrow \infty$.

2) If $z_{1} \neq 0$ then the process

$$
\bar{b}_{T}=\frac{\left\langle Q z_{1}, z_{1}\right\rangle_{\operatorname{Dom}\left((-A)^{\frac{1}{2}}\right)}}{4 a J_{T}-\left\langle Q z_{2}, z_{2}\right\rangle_{L^{2}(D)}}
$$

is strongly consistent estimator of the parameter $b$, i.e., $\bar{b}_{T} \rightarrow b, \mathbb{P}-$ a.s. as $T \rightarrow \infty$. 
Proof. From (3.1) and (2.4) it follows that

$$
\begin{aligned}
\lim _{T \rightarrow \infty} J_{T} & =\left\langle Q_{\infty}^{(a, b)} z, z\right\rangle_{V}=\left\langle\left(\begin{array}{cc}
\frac{1}{4 a b} Q & 0 \\
0 & \frac{1}{4 a} Q
\end{array}\right)\left(\begin{array}{c}
z_{1} \\
z_{2}
\end{array}\right),\left(\begin{array}{c}
z_{1} \\
z_{2}
\end{array}\right)\right\rangle_{V} \\
& =\frac{1}{4 a b}\left\langle Q z_{1}, z_{1}\right\rangle_{\operatorname{Dom}\left((-A)^{\frac{1}{2}}\right)}+\frac{1}{4 a}\left\langle Q z_{2}, z_{2}\right\rangle_{L^{2}(D)}, \quad \mathbb{P}-a . s .
\end{aligned}
$$

Hence we obtain the desired convergence $\bar{a}_{T} \rightarrow a, \mathbb{P}-$ a.s. as $T \rightarrow \infty$ unless $z=0$. Similarly, if $z_{1} \neq 0$ then we obtain the convergence $\bar{b}_{T} \rightarrow b, \mathbb{P}-$ a.s. as $T \rightarrow \infty$.

The estimators $\bar{a}_{T}$ and $\bar{b}_{T}$ have one major disadvantage: In order to compute the estimator $\bar{a}_{T}$, we have to know the true value of the other parameter $b$ (and vice versa for the estimator $\bar{b}_{T}$ ). This problem may be overcome by a more specific choice of the "observation window". Therefore, consider the following special cases or estimation strategies:

1. If $z=\left(0, z_{2}\right)^{\top}$, i.e., $z_{1}=0, z_{2} \neq 0$, then

$$
\bar{a}_{T}=\frac{\left\langle Q z_{2}, z_{2}\right\rangle_{L^{2}(D)}}{\frac{4}{T} \int_{0}^{T}\left\langle X_{2}^{x_{0}}(t), z_{2}\right\rangle_{L^{2}(D)}^{2} d t},
$$

where $X^{x_{0}}(t)=\left(X_{1}^{x_{0}}(t), X_{2}^{x_{0}}(t)\right)^{\top} \in V$ is the solution to the equation (2.2). In order to make such an estimator, only the observation of the second component of the solution is needed.

2. If $z=\left(z_{1}, 0\right)^{\top}$, i.e., $z_{2}=0, z_{1} \neq 0$, then we have that

$$
\lim _{T \rightarrow \infty} J_{T}=\lim _{T \rightarrow \infty} \frac{1}{T} \int_{0}^{T}\left\langle X_{1}^{x_{0}}(t), z_{1}\right\rangle_{\operatorname{Dom}\left((-A)^{\frac{1}{2}}\right)}^{2} d t=\frac{1}{4 a b}\left\langle Q z_{1}, z_{1}\right\rangle_{\operatorname{Dom}\left((-A)^{\frac{1}{2}}\right)}
$$

and it is possible to estimate either the product $a b$, or one of the parameters if the true value of the other one is known. (In this case the formulae (3.2) and (3.3) actually coincide.)

3. It is possible to combine the two previous strategies together. First, using the "window" $z=\left(0, z_{2}\right)^{\top}, z_{2} \neq 0$, we get an estimator of $a$, that is

$$
\bar{a}_{T}=\frac{\left\langle Q z_{2}, z_{2}\right\rangle_{L^{2}(D)}}{\frac{4}{T} \int_{0}^{T}\left\langle X_{2}^{x_{0}}(t), z_{2}\right\rangle_{L^{2}(D)}^{2} d t}
$$

and then, using the "window" $z=\left(z_{1}, 0\right)^{\top}, z_{1} \neq 0$, we get an estimator of $b$, that is

$$
\bar{b}_{T}=\frac{\left\langle Q z_{1}, z_{1}\right\rangle_{\operatorname{Dom}\left((-A)^{\frac{1}{2}}\right)}}{\left\langle Q z_{2}, z_{2}\right\rangle_{L^{2}(D)}} \cdot \frac{\frac{1}{T} \int_{0}^{T}\left\langle X_{2}^{x_{0}}(t), z_{2}\right\rangle_{L^{2}(D)}^{2} d t}{\frac{1}{T} \int_{0}^{T}\left\langle X_{1}^{x_{0}}(t), z_{1}\right\rangle_{\operatorname{Dom}\left((-A)^{\frac{1}{2}}\right)}^{2} d t} .
$$

4. It is also possible to generalize the previous procedure further. First, using the "window" $z=\left(0, z_{2}\right)^{\top}, z_{2} \neq 0$, we get an estimator of $a$, that is

$$
\bar{a}_{T}=\frac{\left\langle Q z_{2}, z_{2}\right\rangle_{L^{2}(D)}}{\frac{4}{T} \int_{0}^{T}\left\langle X_{2}^{x_{0}}(t), z_{2}\right\rangle_{L^{2}(D)}^{2} d t}
$$


and then, using any "window" $\tilde{z}=\left(\tilde{z}_{1}, \tilde{z}_{2}\right)^{\top}, \tilde{z}_{1} \neq 0$, we get an estimator of $b$, that is

$$
\begin{aligned}
\bar{b}_{T}= & \frac{\left\langle Q \tilde{z}_{1}, \tilde{z}_{1}\right\rangle_{\operatorname{Dom}\left((-A)^{\frac{1}{2}}\right)}}{4 \bar{a}_{T} \frac{1}{T} \int_{0}^{T}\left\langle X^{x_{0}}(t), \tilde{z}\right\rangle_{V}^{2} d t-\left\langle Q \tilde{z}_{2}, \tilde{z}_{2}\right\rangle_{L^{2}(D)}} \\
= & \frac{\left\langle Q \tilde{z}_{1}, \tilde{z}_{1}\right\rangle_{\operatorname{Dom}\left((-A)^{\frac{1}{2}}\right)}}{\left\langle Q z_{2}, z_{2}\right\rangle_{L^{2}(D)} \frac{\frac{1}{T} \int_{0}^{T}\left\langle X^{x_{0}}(t), \tilde{z}\right\rangle_{V}^{2} d t}{\frac{1}{T} \int_{0}^{T}\left\langle X_{2}^{x_{0}}(t), z_{2}\right\rangle_{L^{2}(D)}^{2} d t}-\left\langle Q \tilde{z}_{2}, \tilde{z}_{2}\right\rangle_{L^{2}(D)}} \\
= & \frac{\left\langle Q \tilde{z}_{1}, \tilde{z}_{1}\right\rangle_{\operatorname{Dom}\left((-A)^{\frac{1}{2}}\right)} \frac{1}{T} \int_{0}^{T}\left\langle X_{2}^{x_{0}}(t), z_{2}\right\rangle_{L^{2}(D)}^{2} d t}{\left\langle Q z_{2}, z_{2}\right\rangle_{L^{2}(D)} \frac{1}{T} \int_{0}^{T}\left\langle X^{x_{0}}(t), \tilde{z}\right\rangle_{V}^{2} d t-\left\langle Q \tilde{z}_{2}, \tilde{z}_{2}\right\rangle_{L^{2}(D)} \frac{1}{T} \int_{0}^{T}\left\langle X_{2}^{x_{0}}(t), z_{2}\right\rangle_{L^{2}(D)}^{2} d t} .
\end{aligned}
$$

For the practical reasons there is an incentive to observe the solution to the equation (2.2) through the "observation window" componentwise (i.e., we observe the processes $\left(\left\langle X_{1}^{x_{0}}, z_{1}\right\rangle_{\operatorname{Dom}\left((-A)^{\frac{1}{2}}\right)}, 0 \leqslant t \leqslant T\right)$ and $\left(\left\langle X_{2}^{x_{0}}, z_{2}\right\rangle_{L^{2}(D)}, 0 \leqslant t \leqslant T\right)$ for any given $0 \neq z_{1} \in V, 0 \neq z_{2} \in \operatorname{Dom}\left((-A)^{\frac{1}{2}}\right)$ separately), so we will prefer the strategy 3 . Let us introduce these estimators once again with the new notation.

Corollary 3.3. 1) Let $0 \neq z_{2} \in L^{2}(D)$ be arbitrary. The process

$$
\bar{a}_{T, z_{2}}=\frac{\left\langle Q z_{2}, z_{2}\right\rangle_{L^{2}(D)}}{\frac{4}{T} \int_{0}^{T}\left\langle X_{2}^{x_{0}}(t), z_{2}\right\rangle_{L^{2}(D)}^{2} d t}
$$

is strongly consistent estimator of the parameter $a$.

2) Moreover, let $0 \neq z_{1} \in \operatorname{Dom}\left((-A)^{\frac{1}{2}}\right)$ be arbitrary. The process

$$
\bar{b}_{T, z_{1}, z_{2}}=\frac{\left\langle Q z_{1}, z_{1}\right\rangle_{\operatorname{Dom}\left((-A)^{\frac{1}{2}}\right)}}{\left\langle Q z_{2}, z_{2}\right\rangle_{L^{2}(D)}} \cdot \frac{\frac{1}{T} \int_{0}^{T}\left\langle X_{2}^{x_{0}}(t), z_{2}\right\rangle_{L^{2}(D)}^{2} d t}{\frac{1}{T} \int_{0}^{T}\left\langle X_{1}^{x_{0}}(t), z_{1}\right\rangle_{\operatorname{Dom}\left((-A)^{\frac{1}{2}}\right)}^{2} d t}
$$

is strongly consistent estimator of the parameter $b$.

Proof. It is the direct consequence of Theorem 3.2

The previous estimators may be specified even further if the "observation window" is the element of the orthonormal basis.

Indeed, if $z_{2}=e_{k} \in L^{2}(D)$ for any $k \in \mathbb{N}$ then $\bar{a}_{T, z_{2}}$ takes the form

$$
\bar{a}_{T, k}:=\bar{a}_{T, e_{k}}=\frac{\lambda_{k}}{\frac{4}{T} \int_{0}^{T}\left\langle X_{2}^{x_{0}}(t), e_{k}\right\rangle_{L^{2}(D)}^{2} d t} .
$$

Moreover, if $z_{1}=f_{j} \in \operatorname{Dom}\left((-A)^{\frac{1}{2}}\right)$ for any $j \in \mathbb{N}$ then $\bar{b}_{T, z_{1}, z_{2}}$ takes the form

$$
\bar{b}_{T, j, k}:=\bar{b}_{T, f_{j}, e_{k}}=\frac{\lambda_{j}}{\lambda_{k}} \cdot \frac{\frac{1}{T} \int_{0}^{T}\left\langle X_{2}^{x_{0}}(t), e_{k}\right\rangle_{L^{2}(D)}^{2} d t}{\frac{1}{T}\left\langle X_{1}^{x_{0}}(t), f_{j}\right\rangle_{\operatorname{Dom}\left((-A)^{\frac{1}{2}}\right)}^{2} d t} .
$$

These estimators are using only observation of some given modes in the expansion of the solution. 


\section{Asymptotic normality of the estimators}

4.1. Asymptotic normality of the estimator $\bar{a}_{T, z_{2}}$. In this section we show asymptotic normality of the estimator (3.4), i.e., the weak convergence of $\sqrt{T}\left(\bar{a}_{T, z_{2}}-\right.$ a) to a Gaussian distribution.

Let $k \in \mathbb{N}$ be arbitrary and define the operator $E_{k}: V \rightarrow V$ by

$$
E_{k} x=E_{k}\left(\begin{array}{l}
x_{1} \\
x_{2}
\end{array}\right)=\left(\begin{array}{ll}
E_{k, 1} & E_{k, 2} \\
E_{k, 3} & E_{k, 4}
\end{array}\right)\left(\begin{array}{c}
x_{1} \\
x_{2}
\end{array}\right), \quad \forall x=\left(\begin{array}{c}
x_{1} \\
x_{2}
\end{array}\right) \in V,
$$

where $E_{k, 2}=0, E_{k, 3}=0$ and

$$
\begin{aligned}
& E_{k, 1}: x_{1} \longmapsto b\left\langle x_{1}, e_{k}\right\rangle_{L^{2}(D)} e_{k}, \\
& E_{k, 4}: x_{2} \longmapsto\left\langle x_{2}, e_{k}\right\rangle_{L^{2}(D)} e_{k},
\end{aligned}
$$

for any $x_{1} \in \operatorname{Dom}\left((-A)^{\frac{1}{2}}\right)$ and $x_{2} \in L^{2}(D)$. Hence the operator $E_{k}$ evaluates as

$$
E_{k} x=\left(\begin{array}{c}
b\left\langle x_{1}, e_{k}\right\rangle_{L^{2}(D)} e_{k} \\
\left\langle x_{2}, e_{k}\right\rangle_{L^{2}(D)} e_{k}
\end{array}\right), \quad \forall x=\left(\begin{array}{c}
x_{1} \\
x_{2}
\end{array}\right) \in V .
$$

Let $k, l \in \mathbb{N}$ be arbitrary and define the operator $E_{k, l}: V \rightarrow V$ by

$$
E_{k, l} x=E_{k, l}\left(\begin{array}{c}
x_{1} \\
x_{2}
\end{array}\right)=\frac{1}{D_{k, l}}\left(\begin{array}{cc}
E_{k, l, 1} & E_{k, l, 2} \\
E_{k, l, 3} & E_{k, l, 4}
\end{array}\right)\left(\begin{array}{c}
x_{1} \\
x_{2}
\end{array}\right), \quad \forall x=\left(\begin{array}{c}
x_{1} \\
x_{2}
\end{array}\right) \in V,
$$

where

$$
\begin{aligned}
& E_{k, l, 1}: x_{1} \longmapsto 16 a^{2} b \alpha_{l}\left\langle x_{1}, e_{l}\right\rangle_{L^{2}(D)} e_{k}+16 a^{2} b \alpha_{k}\left\langle x_{1}, e_{k}\right\rangle_{L^{2}(D)} e_{l}, \\
& E_{k, l, 2}: x_{2} \longmapsto 4 a b\left(\alpha_{k}-\alpha_{l}\right)\left\langle x_{2}, e_{k}\right\rangle_{L^{2}(D)} e_{l}+4 a b\left(\alpha_{l}-\alpha_{k}\right)\left\langle x_{2}, e_{l}\right\rangle_{L^{2}(D)} e_{k}, \\
& E_{k, l, 3}: x_{1} \longmapsto 4 a b \alpha_{l}\left(\alpha_{k}-\alpha_{l}\right)\left\langle x_{1}, e_{l}\right\rangle_{L^{2}(D)} e_{k}+4 a b \alpha_{k}\left(\alpha_{l}-\alpha_{k}\right)\left\langle x_{1}, e_{k}\right\rangle_{L^{2}(D)} e_{l}, \\
& E_{k, l, 4}: x_{2} \longmapsto 8 a^{2}\left(\alpha_{k}+\alpha_{l}\right)\left\langle x_{2}, e_{k}\right\rangle_{L^{2}(D)} e_{l}+8 a^{2}\left(\alpha_{k}+\alpha_{l}\right)\left\langle x_{2}, e_{l}\right\rangle_{L^{2}(D)} e_{k},
\end{aligned}
$$

for any $x_{1} \in \operatorname{Dom}\left((-A)^{\frac{1}{2}}\right), x_{2} \in L^{2}(D)$ and $D_{k, l}$ defined by

$$
D_{k, l}=b\left(\alpha_{k}-\alpha_{l}\right)^{2}+8 a^{2}\left(\alpha_{k}+\alpha_{l}\right) .
$$

Note that $D_{k, l}$ is the denominator from the formula (2.3) divided by $b$ and that $D_{k, l}=D_{l, k}$. Also note that $D_{k, k}=16 a^{2} \alpha_{k}$.

The properties of the operators $E_{k}$ and $E_{k, l}$ are summarized in the following Lemma.

Lemma 4.1. 1) The operator $E_{k} \in \mathcal{L}(V)$ is self-adjoint for any given $k \in \mathbb{N}$. Moreover,

$$
\left\langle E_{k} x, \mathcal{A} x\right\rangle_{V}=-2 a\left\langle x_{2}, e_{k}\right\rangle_{L^{2}(D)}^{2}, \quad \forall x=\left(\begin{array}{l}
x_{1} \\
x_{2}
\end{array}\right) \in \operatorname{Dom}(\mathcal{A}) .
$$

2) The operator $E_{k, l} \in \mathcal{L}(V)$ is self-adjoint for any given $k, l \in \mathbb{N}$. Moreover,

$$
\left\langle E_{k, l} x, \mathcal{A} x\right\rangle_{V}=-4 a\left\langle x_{2}, e_{k}\right\rangle_{L^{2}(D)}\left\langle x_{2}, e_{l}\right\rangle_{L^{2}(D)}, \quad \forall x=\left(\begin{array}{c}
x_{1} \\
x_{2}
\end{array}\right) \in \operatorname{Dom}(\mathcal{A}) .
$$


Proof. 1) Let $k \in \mathbb{N}$ be arbitrary. It is evident that $E_{k} \in \mathcal{L}(V)$ and for $x=$ $\left(x_{1}, x_{2}\right)^{\top} \in V$ and $y=\left(y_{1}, y_{2}\right)^{\top} \in V$ we have

$$
\begin{aligned}
\left\langle E_{k} x, y\right\rangle_{V} & =\left\langle\left(\begin{array}{c}
b\left\langle x_{1}, e_{k}\right\rangle_{L^{2}(D)} e_{k} \\
\left\langle x_{2}, e_{k}\right\rangle_{L^{2}(D)} e_{k}
\end{array}\right),\left(\begin{array}{c}
y_{1} \\
y_{2}
\end{array}\right)\right\rangle_{V} \\
& =b\left\langle x_{1}, e_{k}\right\rangle_{L^{2}(D)}\left\langle y_{1}, e_{k}\right\rangle_{\operatorname{Dom}\left((-A)^{\frac{1}{2}}\right)}+\left\langle x_{2}, e_{k}\right\rangle_{L^{2}(D)}\left\langle y_{2}, e_{k}\right\rangle_{L^{2}(D)} \\
& =b \alpha_{k}\left\langle x_{1}, e_{k}\right\rangle_{L^{2}(D)}\left\langle y_{1}, e_{k}\right\rangle_{L^{2}(D)}+\left\langle x_{2}, e_{k}\right\rangle_{L^{2}(D)}\left\langle y_{2}, e_{k}\right\rangle_{L^{2}(D)} \\
& =\left\langle x, E_{k} y\right\rangle_{V},
\end{aligned}
$$

hence $E_{k}=E_{k}^{*}$. Moreover, for every $x=\left(x_{1}, x_{2}\right)^{\top} \in \operatorname{Dom}(\mathcal{A})$ we have

$$
\begin{aligned}
\left\langle E_{k} x, \mathcal{A} x\right\rangle_{V}= & \left\langle\left(\begin{array}{c}
b\left\langle x_{1}, e_{k}\right\rangle_{L^{2}(D)} e_{k} \\
\left\langle x_{2}, e_{k}\right\rangle_{L^{2}(D)} e_{k}
\end{array}\right),\left(\begin{array}{c}
x_{2} \\
b A x_{1}-2 a x_{2}
\end{array}\right)\right\rangle_{V} \\
= & b\left\langle x_{1}, e_{k}\right\rangle_{L^{2}(D)}\left\langle(-A)^{\frac{1}{2}} x_{2},(-A)^{\frac{1}{2}} e_{k}\right\rangle_{L^{2}(D)} \\
& +b\left\langle x_{2}, e_{k}\right\rangle_{L^{2}(D)}\left\langle A x_{1}, e_{k}\right\rangle_{L^{2}(D)}-2 a\left\langle x_{2}, e_{k}\right\rangle_{L^{2}(D)}^{2} \\
= & -2 a\left\langle x_{2}, e_{k}\right\rangle_{L^{2}(D)}^{2} .
\end{aligned}
$$

2) Let $k, l \in \mathbb{N}$ be arbitrary. It is evident that $E_{k, l} \in \mathcal{L}(V)$ and similarly as above it is possible to verify that $E_{k, l}=E_{k, l}^{*}$ and that (4.2) holds true for any $x \in \operatorname{Dom}(\mathcal{A})$.

Choose $0 \neq z_{2} \in L^{2}(D)$ taking the form

$$
z_{2}=\sum_{k=1}^{\infty}\left\langle z_{2}, e_{k}\right\rangle_{L^{2}(D)} e_{k}=\sum_{k=1}^{\infty} z_{2, k} e_{k}
$$

Finally, define the operator $E: V \rightarrow V$ by

$$
E=\sum_{k=1}^{\infty} z_{2, k}^{2} E_{k}+\sum_{k=1}^{\infty} \sum_{l=k+1}^{\infty} z_{2, k} z_{2, l} E_{k, l}
$$

The properties of the operator $E$ needed in the sequel are summarized in the following Lemma.

Lemma 4.2. The operator $E \in \mathcal{L}(V)$. Moreover, it is self-adjoint and

$$
\langle E x, \mathcal{A} x\rangle_{V}=-2 a\left\langle x_{2}, z_{2}\right\rangle_{L^{2}(D)}^{2}, \quad \forall x=\left(\begin{array}{l}
x_{1} \\
x_{2}
\end{array}\right) \in \operatorname{Dom}(\mathcal{A}) .
$$

Proof. There exists a positive constant $C>0$ (which does not depend on $k$ ) such that $\left\|E_{k}\right\|_{\mathcal{L}(V)}<C$ for any $k \in \mathbb{N}$. Hence

$$
\left\|\sum_{k=1}^{\infty} z_{2, k}^{2} E_{k}\right\|_{\mathcal{L}(V)} \leqslant \sum_{k=1}^{\infty} z_{2, k}^{2}\left\|E_{k}\right\|_{\mathcal{L}(V)} \leqslant C \sum_{k=1}^{\infty} z_{2, k}^{2}=C\left\|z_{2}\right\|_{L^{2}(D)}^{2}<\infty
$$

and the operator defined by the first sum in (4.3) belongs to the space $\mathcal{L}(V)$.

The convergence of the double series is fulfilled by the denominator $D_{k, l}$. For any $k, l \in \mathbb{N}$ we have

$$
\frac{\alpha_{k}+\alpha_{l}}{D_{k, l}} \leqslant \frac{\alpha_{k}+\alpha_{l}}{8 a^{2}\left(\alpha_{k}+\alpha_{l}\right)}=\frac{1}{8 a}
$$




$$
\begin{aligned}
\frac{\sqrt{\alpha_{k}}\left|\alpha_{k}-\alpha_{l}\right|}{D_{k, l}} & =\sqrt{\frac{\alpha_{k}}{D_{k, l}}} \frac{\left|\alpha_{k}-\alpha_{l}\right|}{\sqrt{D_{k, l}}}<\frac{1}{\sqrt{8 a^{2}}} \frac{\left|\alpha_{k}-\alpha_{l}\right|}{\sqrt{b\left(\alpha_{k}-\alpha_{l}\right)^{2}+8 a^{2}\left(\alpha_{k}+\alpha_{l}\right)}} \\
& <\frac{1}{\sqrt{8 a^{2}}} \frac{\left|\alpha_{k}-\alpha_{l}\right|}{\sqrt{b}\left|\alpha_{k}-\alpha_{l}\right|}=\frac{1}{8 a^{2} b}, \\
& \frac{\sqrt{\alpha_{k} \alpha_{l}}}{D_{k, l}}<\frac{2 \sqrt{\alpha_{k} \alpha_{l}}}{D_{k, l}} \leqslant \frac{2 \sqrt{\alpha_{k} \alpha_{l}}}{8 a^{2}\left(\alpha_{k}+\alpha_{l}\right)} \leqslant \frac{1}{8 a^{2}} .
\end{aligned}
$$

The desired convergence is then accomplished by the convergence of the series

$$
\sum_{k=1}^{\infty} z_{2, k}^{2}<\infty, \quad \sum_{k=1}^{\infty}\left\langle x_{2}, e_{k}\right\rangle_{L^{2}(D)}^{2}<\infty, \quad \sum_{k=1}^{\infty} \alpha_{k}\left\langle x_{1}, e_{k}\right\rangle_{L^{2}(D)}^{2}<\infty .
$$

The linear combination of the self-adjoint operators is also the self-adjoint operator, hence $E=E^{*}$.

The property (4.4) follows by (4.1), (4.2) and the following computation

$$
\begin{aligned}
& \langle E x, \mathcal{A} x\rangle_{V}= \\
& =\left\langle\left(\sum_{k=1}^{\infty} z_{2, k}^{2} E_{k}+\sum_{k=1}^{\infty} \sum_{l=k+1}^{\infty} z_{2, k} z_{2, l} E_{k, l}\right) x, \mathcal{A} x\right\rangle_{V} \\
& =\sum_{k=1}^{\infty} z_{2, k}^{2}\left\langle E_{k} x, \mathcal{A} x\right\rangle_{V}+\sum_{k=1}^{\infty} \sum_{l=k+1}^{\infty} z_{2, k} z_{2, l}\left\langle E_{k, l} x, \mathcal{A} x\right\rangle_{V} \\
& =-2 a\left(\sum_{k=1}^{\infty} z_{2, k}^{2}\left\langle x_{2}, e_{k}\right\rangle_{L^{2}(D)}^{2}+2 \sum_{k=1}^{\infty} \sum_{l=k+1}^{\infty} z_{2, k} z_{2, l}\left\langle x_{2}, e_{k}\right\rangle_{L^{2}(D)}\left\langle x_{2}, e_{l}\right\rangle_{L^{2}(D)}\right) \\
& =-2 a\left(\sum_{k=1}^{\infty} z_{2, k}\left\langle x_{2}, e_{k}\right\rangle_{L^{2}(D)}\right)^{2} \\
& =-2 a\left\langle x_{2}, \sum_{k=1}^{\infty} z_{2, k} e_{k}\right\rangle_{L^{2}(D)}^{2} \\
& =-2 a\left\langle x_{2}, z_{2}\right\rangle_{L^{2}(D)}^{2} .
\end{aligned}
$$

We will need an alternative representation for the process $\frac{1}{T} \int_{0}^{T}\left\langle X_{2}^{x_{0}}(t), z_{2}\right\rangle_{L^{2}(D)}^{2} d t$

Lemma 4.3. The process $\frac{1}{T} \int_{0}^{T}\left\langle X_{2}^{x_{0}}(t), z_{2}\right\rangle_{L^{2}(D)}^{2} d t$ admits the following representation

$$
\begin{aligned}
\frac{1}{T} \int_{0}^{T}\left\langle X_{2}^{x_{0}}(t), z_{2}\right\rangle_{L^{2}(D)}^{2} d t= & -\frac{1}{4 a T}\left(\left\langle E X^{x_{0}}(T), X^{x_{0}}(T)\right\rangle_{V}-\left\langle E x_{0}, x_{0}\right\rangle_{V}\right) \\
& +\frac{1}{2 a T} \int_{0}^{T}\left\langle E X^{x_{0}}(t), \Phi d B(t)\right\rangle_{V}+\frac{1}{4 a}\left\langle Q z_{2}, z_{2}\right\rangle_{L^{2}(D)} .
\end{aligned}
$$

Proof. Define the function $g: V \rightarrow \mathbb{R}$ by

$$
g(x)=\langle E x, x\rangle_{V}, \quad \forall x \in V .
$$


The Itô's formula (see e.g. 11, Theorem 4.17.) is not applicable to the process $g\left(X^{x_{0}}(t)\right)$ directly, because $\left(X^{x_{0}}(t), t \geqslant 0\right)$ is not a strong solution to the equation (2.2). We apply it to suitable finite-dimensional projections.

Let $\left\{h_{n}, n \in \mathbb{N}\right\}$ be an orthonormal basis in $V$ consisting of elements from $\operatorname{Dom}(\mathcal{A})$ and let $P_{N}$ be the operator of projection on the $\operatorname{span}\left\{h_{n}, n=1, \ldots N\right\}$, that is

$$
P_{N} x=\sum_{n=1}^{N}\left\langle x, h_{n}\right\rangle_{V} h_{n}, \quad \forall x \in V, \quad \forall N \in \mathbb{N} .
$$

Choose $N \in \mathbb{N}$ and set

$$
X^{x_{0}, N}(t):=P_{N} X^{x_{0}}(t), \quad \forall t \geqslant 0 .
$$

The expansion for the $X^{x_{0}, N}(t)$ is finite, so $X_{1}^{x_{0}, N}(t) \in \operatorname{Dom}(A), X_{2}^{x_{0}, N}(t) \in$ $\operatorname{Dom}\left((-A)^{\frac{1}{2}}\right)$ and consequently $X^{x_{0}, N}(t) \in \operatorname{Dom}(\mathcal{A})$ for all $t \geqslant 0$. Now we may apply Itô's formula to the function $g\left(X^{x_{0}, N}(t)\right)$, which yields

$$
d g\left(X^{x_{0}, N}(t)\right)=2\left\langle E X^{x_{0}, N}(t), d X^{x_{0}, N}(t)\right\rangle_{V}+\frac{1}{2} \operatorname{Tr}\left(2 E \Phi \Phi^{*}\right) d t
$$

where $\operatorname{Tr}(\cdot)$ denotes the trace of the (nuclear) operator.

First, we simplify the second term by the following calculation

$$
\begin{aligned}
\operatorname{Tr}\left(E \Phi \Phi^{*}\right) & =\operatorname{Tr}\left(\left(\begin{array}{cc}
E_{1} & E_{2} \\
E_{3} & E_{4}
\end{array}\right)\left(\begin{array}{cc}
0 & 0 \\
0 & Q
\end{array}\right)\right)=\operatorname{Tr}\left(\begin{array}{cc}
0 & E_{2} Q \\
0 & E_{4} Q
\end{array}\right)=\operatorname{Tr}\left(E_{4} Q\right) \\
& =\operatorname{Tr}\left(\sum_{k=1}^{\infty} z_{2, k}^{2} E_{k, 4} Q+\sum_{k=1}^{\infty} \sum_{l=k+1}^{\infty} \frac{z_{2, k} z_{2, l}}{D_{k, l}} E_{k, l, 4} Q\right) \\
& =\sum_{k=1}^{\infty} z_{2, k}^{2} \operatorname{Tr}\left(E_{k, 4} Q\right)+\sum_{k=1}^{\infty} \sum_{l=k+1}^{\infty} \frac{z_{2, k} z_{2, l}}{D_{k, l}} \operatorname{Tr}\left(E_{k, l, 4} Q\right) .
\end{aligned}
$$

Now we compute the partial traces $\operatorname{Tr}\left(E_{k, 4} Q\right)$ and $\operatorname{Tr}\left(E_{k, l, 4} Q\right)$. According to the definition of the trace

$$
\begin{aligned}
\operatorname{Tr}\left(E_{k, 4} Q\right) & =\sum_{j=1}^{\infty}\left\langle E_{k, 4} Q e_{j}, e_{j}\right\rangle_{L^{2}(D)}=\sum_{j=1}^{\infty} \lambda_{j}\left\langle E_{k, 4} e_{j}, e_{j}\right\rangle_{L^{2}(D)} \\
& =\sum_{j=1}^{\infty} \lambda_{j}\left\langle e_{j}, e_{k}\right\rangle_{L^{2}(D)}^{2}=\sum_{j=1}^{\infty} \lambda_{j} \delta_{j, k}=\lambda_{k},
\end{aligned}
$$

where $\delta$ stands for the Kronecker's delta. Similarly, we have

$$
\begin{aligned}
\operatorname{Tr}\left(E_{k, l, 4} Q\right)= & \sum_{j=1}^{\infty}\left\langle E_{k, l, 4} Q e_{j}, e_{j}\right\rangle_{L^{2}(D)} \\
= & \sum_{j=1}^{\infty} \lambda_{j}\left(8 a^{2}\left(\alpha_{k}+\alpha_{l}\right)\left\langle e_{j}, e_{k}\right\rangle_{L^{2}(D)}\left\langle e_{l}, e_{j}\right\rangle_{L^{2}(D)}\right. \\
& \left.+8 a^{2}\left(\alpha_{k}+\alpha_{l}\right)\left\langle e_{j}, e_{l}\right\rangle_{L^{2}(D)}\left\langle e_{k}, e_{j}\right\rangle_{L^{2}(D)}\right) \\
= & \sum_{j=1}^{\infty} 16 a^{2} \lambda_{j}\left(\alpha_{k}+\alpha_{l}\right) \delta_{j, k} \delta_{j, l}=0
\end{aligned}
$$


since $k \neq l$ in (4.3). Therefore

$$
\operatorname{Tr}\left(E \Phi \Phi^{*}\right)=\sum_{k=1}^{\infty} \lambda_{k} z_{2, k}^{2}=\left\langle Q z_{2}, z_{2}\right\rangle_{L^{2}(D)} .
$$

Using this formulae and Lemma 4.2 the expression (4.6) implies

$$
\begin{aligned}
d g\left(X^{x_{0}, N}(t)\right)= & 2\left\langle E X^{x_{0}, N}(t), \mathcal{A} X^{x_{0}, N}(t)\right\rangle_{V} d t+2\left\langle E X^{x_{0}, N}(t), \Phi d B(t)\right\rangle_{V} \\
& +\left\langle Q z_{2}, z_{2}\right\rangle_{L^{2}(D)} d t \\
= & -4 a\left\langle X_{2}^{x_{0}, N}(t), z_{2}\right\rangle_{L^{2}(D)}^{2} d t+2\left\langle E X^{x_{0}, N}(t), \Phi d B(t)\right\rangle_{V} \\
& +\left\langle Q z_{2}, z_{2}\right\rangle_{L^{2}(D)} d t .
\end{aligned}
$$

Integrating previous formula over the interval $(0, T)$, we arrive at

$$
\begin{aligned}
& \frac{1}{T} \int_{0}^{T}\left\langle X_{2}^{x_{0}, N}(t), z_{2}\right\rangle_{L^{2}(D)}^{2} d t= \\
& =-\frac{1}{4 a T}\left(\left\langle E X^{x_{0}, N}(T), X^{x_{0}, N}(T)\right\rangle_{V}-\left\langle E x_{0}^{N}, x_{0}^{N}\right\rangle_{V}\right) \\
& \quad+\frac{1}{2 a T} \int_{0}^{T}\left\langle E X^{x_{0}, N}(t), \Phi d B(t)\right\rangle_{V}+\frac{1}{4 a}\left\langle Q z_{2}, z_{2}\right\rangle_{L^{2}(D)} .
\end{aligned}
$$

Since

$$
\left|\left\langle X_{2}^{x_{0}, N}(t), z_{2}\right\rangle_{L^{2}(D)}\right| \leqslant\left\|X_{2}^{x_{0}}(t)\right\|_{L^{2}(D)}\left\|z_{2}\right\|_{L^{2}(D)}, \quad \forall t \geqslant 0, \quad \forall N \in \mathbb{N},
$$

we may use the random variable $\left\|X_{2}^{x_{0}}(t)\right\|_{L^{2}(D)}^{2}$ as an integrable majorant for the integral on the left-hand side. Also,

$$
\int_{0}^{T}\left\langle E X^{x_{0}, N}(t), \Phi d B(t)\right\rangle_{V} \rightarrow \int_{0}^{T}\left\langle E X^{x_{0}}(t), \Phi d B(t)\right\rangle_{V}, \quad N \rightarrow \infty \text { in } L^{2}(\Omega),
$$

because, for some positive constant $C>0$, we have that

$\mathbb{E}\left|\int_{0}^{T}\left\langle E\left(X^{x_{0}, N}(t)-X^{x_{0}}(t)\right), \Phi d B(t)\right\rangle_{V}\right|^{2} \leqslant C \int_{0}^{T} \mathbb{E}\left\|X^{x_{0}, N}(t)-X^{x_{0}}(t)\right\|_{V}^{2} d t$,

which tends to 0 as $N \rightarrow \infty$, since

$$
X^{x_{0}, N}(t) \rightarrow X^{x_{0}}(t), \quad \forall t \geqslant 0, \quad N \rightarrow \infty \text { in } L^{2}(\Omega ; V) .
$$

Hence we obtain (4.5) by passing $N$ to infinity in (4.7).

We will also need the following Lemma for convergence of some cross terms to zero.

Lemma 4.4. 1) Let $z_{1} \in \operatorname{Dom}\left((-A)^{\frac{1}{2}}\right)$ and $z_{2} \in L^{2}(D)$ be arbitrary. Then

$$
\lim _{T \rightarrow \infty} \frac{1}{T} \int_{0}^{T}\left\langle X_{1}^{x_{0}}(t), z_{1}\right\rangle_{\operatorname{Dom}\left((-A)^{\frac{1}{2}}\right)}\left\langle X_{2}^{x_{0}}(t), z_{2}\right\rangle_{L^{2}(D)} d t=0, \quad \mathbb{P}-\text { a.s. }
$$

2) Let $f_{k}, f_{l} \in \operatorname{Dom}\left((-A)^{\frac{1}{2}}\right), k \neq l$, be arbitrary. Then

$$
\lim _{T \rightarrow \infty} \frac{1}{T} \int_{0}^{T}\left\langle X_{1}^{x_{0}}(t), f_{k}\right\rangle_{\operatorname{Dom}\left((-A)^{\frac{1}{2}}\right)}\left\langle X_{1}^{x_{0}}(t), f_{l}\right\rangle_{\operatorname{Dom}\left((-A)^{\frac{1}{2}}\right)} d t=0, \quad \mathbb{P}-\text { a.s. }
$$


3) Let $e_{k}, e_{l} \in L^{2}(D), k \neq l$, be arbitrary. Then

$$
\lim _{T \rightarrow \infty} \frac{1}{T} \int_{0}^{T}\left\langle X_{2}^{x_{0}}(t), e_{k}\right\rangle_{L^{2}(D)}\left\langle X_{2}^{x_{0}}(t), e_{l}\right\rangle_{L^{2}(D)} d t=0, \quad \mathbb{P}-\text { a.s. }
$$

Proof. 1) Let $z_{1} \in \operatorname{Dom}\left((-A)^{\frac{1}{2}}\right)$ and $z_{2} \in L^{2}(D)$. Using Theorem 3.1 with a functional $\varrho: V \rightarrow \mathbb{R}, \varrho(y)=\left\langle y,\left(\begin{array}{c}z_{1} \\ 0\end{array}\right)\right\rangle_{V}\left\langle y,\left(\begin{array}{c}0 \\ z_{2}\end{array}\right)\right\rangle_{V}, y \in V$ and $m=1$, we get

$$
\begin{aligned}
& \lim _{T \rightarrow \infty} \frac{1}{T} \int_{0}^{T}\left\langle X_{1}^{x_{0}}(t), z_{1}\right\rangle_{\operatorname{Dom}\left((-A)^{\frac{1}{2}}\right)}\left\langle X_{2}^{x_{0}}(t), z_{2}\right\rangle_{L^{2}(D)} d t= \\
& =\lim _{T \rightarrow \infty} \frac{1}{T} \int_{0}^{T}\left\langle X^{x_{0}}(t),\left(\begin{array}{c}
z_{1} \\
0
\end{array}\right)\right\rangle_{V}\left\langle X^{x_{0}}(t),\left(\begin{array}{c}
0 \\
z_{2}
\end{array}\right)\right\rangle_{V} d t \\
& =\int_{V}\left\langle y,\left(\begin{array}{c}
z_{1} \\
0
\end{array}\right)\right\rangle_{V}\left\langle y,\left(\begin{array}{c}
0 \\
z_{2}
\end{array}\right)\right\rangle_{V} \mu_{\infty}^{(a, b)}(d y) \\
& =\left\langle Q_{\infty}^{(a, b)}\left(\begin{array}{c}
z_{1} \\
0
\end{array}\right),\left(\begin{array}{c}
0 \\
z_{2}
\end{array}\right)\right\rangle_{V} \\
& =\left\langle\left(\begin{array}{cc}
\frac{1}{4 a b} Q & 0 \\
0 & \frac{1}{4 a} Q
\end{array}\right)\left(\begin{array}{c}
z_{1} \\
0
\end{array}\right),\left(\begin{array}{c}
0 \\
z_{2}
\end{array}\right)\right\rangle_{V}=0, \quad \mathbb{P}-\text { a.s. }
\end{aligned}
$$

2) Let $f_{k}, f_{l} \in \operatorname{Dom}\left((-A)^{\frac{1}{2}}\right)$ with $k \neq l$. Using Theorem 3.1 with a functional

$$
\begin{aligned}
\varrho: V \rightarrow \mathbb{R}, \varrho(y)=\left\langle y,\left(\begin{array}{c}
f_{k} \\
0
\end{array}\right)\right\rangle_{V}\left\langle y,\left(\begin{array}{c}
f_{l} \\
0
\end{array}\right)\right\rangle_{V}, y \in V \text { and } m=1 \text {, we get } \\
\lim _{T \rightarrow \infty} \frac{1}{T} \int_{0}^{T}\left\langle X_{1}^{x_{0}}(t), f_{k}\right\rangle_{\operatorname{Dom}\left((-A)^{\frac{1}{2}}\right)}\left\langle X_{1}^{x_{0}}(t), f_{l}\right\rangle_{\operatorname{Dom}\left((-A)^{\frac{1}{2}}\right)} d t= \\
=\lim _{T \rightarrow \infty} \frac{1}{T} \int_{0}^{T}\left\langle X^{x_{0}}(t),\left(\begin{array}{c}
f_{k} \\
0
\end{array}\right)\right\rangle_{V}\left\langle X^{x_{0}}(t),\left(\begin{array}{c}
f_{l} \\
0
\end{array}\right)\right\rangle_{V} d t \\
=\left\langle\left(\begin{array}{cc}
\frac{1}{4 a b} Q & 0 \\
0 & \frac{1}{4 a} Q
\end{array}\right)\left(\begin{array}{c}
f_{k} \\
0
\end{array}\right),\left(\begin{array}{c}
f_{l} \\
0
\end{array}\right)\right\rangle_{V} \\
=\frac{1}{4 a b}\left\langle Q f_{k}, f_{l}\right\rangle_{\operatorname{Dom}\left((-A)^{\frac{1}{2}}\right)} \\
=\frac{\lambda_{k}}{4 a b} \delta_{k, l}=0, \quad \mathbb{P}-a . s .
\end{aligned}
$$

3) Let $e_{k}, e_{l} \in L^{2}(D)$ with $k \neq l$. The proof is analogous to the previous one with functional $\varrho: V \rightarrow \mathbb{R}, \varrho(y)=\left\langle y,\left(\begin{array}{c}0 \\ e_{k}\end{array}\right)\right\rangle_{V}\left\langle y,\left(\begin{array}{c}0 \\ e_{l}\end{array}\right)\right\rangle_{V}, y \in V$.

We will also need the following Lemma from [4].

Lemma 4.5. Let $\left(X^{x_{0}}(t), t \geqslant 0\right)$ be a solution to the linear equation (2.2) and $R \in \mathcal{L}(V)$. Then

$$
\frac{1}{\sqrt{t}}\left\langle R X^{x_{0}}(t), X^{x_{0}}(t)\right\rangle_{V} \rightarrow 0
$$

in $L^{1}(\Omega)$ as $t \rightarrow \infty$.

Proof. See 4 . 
Asymptotic normality of the estimator $\bar{a}_{T, z_{2}}$ is formulated in the following Theorem.

Theorem 4.6. Let $0 \neq z_{2} \in L^{2}(D)$ be arbitrary. The estimator $\bar{a}_{T, z_{2}}$ is asymptotically normal, i.e.,

$$
\begin{aligned}
& \operatorname{Law}\left(\sqrt{T}\left(\bar{a}_{T, z_{2}}-a\right)\right) \stackrel{w^{*}}{\rightarrow} \\
& \quad N\left(0, \frac{8 a^{3}}{\left\langle Q z_{2}, z_{2}\right\rangle_{L^{2}(D)}^{2}} \sum_{k=1}^{\infty} \sum_{n=1}^{\infty} \frac{\lambda_{k} \lambda_{n}\left(\alpha_{k}+\alpha_{n}\right) z_{2, k}^{2} z_{2, n}^{2}}{b\left(\alpha_{k}-\alpha_{n}\right)^{2}+8 a^{2}\left(\alpha_{k}+\alpha_{n}\right)}\right), \quad T \rightarrow \infty .
\end{aligned}
$$

Proof. Set $0 \neq z_{2} \in L^{2}(D)$. If we use formula (3.4) for the estimator $\bar{a}_{T, z_{2}}$ and Lemma 4.3, we obtain

$$
\begin{aligned}
= & \frac{\sqrt{T}}{\frac{4}{T} \int_{0}^{T}\left\langle X_{2}^{x_{0}}(t), z_{2}\right\rangle_{L^{2}(D)}^{2} d t}\left(\left\langle Q z_{2}, z_{2}\right\rangle_{L^{2}(D)}-4 a \frac{1}{T} \int_{0}^{T}\left\langle X_{2}^{x_{0}}(t), z_{2}\right\rangle_{L^{2}(D)}^{2} d t\right) \\
= & \frac{\sqrt{T}}{\frac{4}{T} \int_{0}^{T}\left\langle X_{2}^{x_{0}}(t), z_{2}\right\rangle_{L^{2}(D)}^{2} d t}\left(\frac{1}{T}\left(\left\langle E X^{x_{0}}(T), X^{x_{0}}(T)\right\rangle_{V}-\left\langle E x_{0}, x_{0}\right\rangle_{V}\right)\right. \\
& \left.-\frac{2}{T} \int_{0}^{T}\left\langle E X^{x_{0}}(t), \Phi d B(t)\right\rangle_{V}\right) \\
= & \frac{1}{\frac{4}{T} \int_{0}^{T}\left\langle X_{2}^{x_{0}}(t), z_{2}\right\rangle_{L^{2}(D)}^{2} d t} \frac{1}{\sqrt{T}}\left(\left\langle E X^{x_{0}}(T), X^{x_{0}}(T)\right\rangle_{V}-\left\langle E x_{0}, x_{0}\right\rangle_{V}\right) \\
& -\frac{1}{\frac{2}{T} \int_{0}^{T}\left\langle X_{2}^{x_{0}}(t), z_{2}\right\rangle_{L^{2}(D)}^{2} d t} \frac{1}{\sqrt{T}} \int_{0}^{T}\left\langle E X^{x_{0}}(t), \Phi d B(t)\right\rangle_{V} .
\end{aligned}
$$

The first term on the right-hand side converges to zero in probability as $T \rightarrow \infty$, since

$$
\lim _{T \rightarrow \infty} \frac{1}{T} \int_{0}^{T}\left\langle X_{2}^{x_{0}}(t), z_{2}\right\rangle_{L^{2}(D)}^{2} d t=\frac{1}{4 a}\left\langle Q z_{2}, z_{2}\right\rangle_{L^{2}(D)}, \quad \mathbb{P}-a . s .
$$

by Theorem 3.2 and

$$
\lim _{T \rightarrow \infty} \frac{1}{\sqrt{T}}\left(\left\langle E X^{x_{0}}(T), X^{x_{0}}(T)\right\rangle_{V}-\left\langle E x_{0}, x_{0}\right\rangle_{V}\right)=0, \quad \text { in } L^{1}(\Omega)
$$

by Lemma 4.5 Define

$$
\begin{aligned}
w(T) & =\frac{1}{\sqrt{T}} \int_{0}^{T}\left\langle E X^{x_{0}}(t), \Phi d B(t)\right\rangle_{V} \\
& =\frac{1}{\sqrt{T}} \int_{0}^{T} \sum_{n=1}^{\infty} \sqrt{\lambda_{n}}\left\langle E X^{x_{0}}(t),\left(\begin{array}{c}
0 \\
e_{n}
\end{array}\right)\right\rangle_{V} d \beta_{n}(t),
\end{aligned}
$$

where we have used the representation of $V$-valued Brownian motion $B(t)$. For any $n \in \mathbb{N} \beta_{n}(t)=\left\langle B(t), e_{n}\right\rangle_{V}$ are mutually independent scalar Brownian motions (see [1]).

First, let us express scalar product in the above series

$$
\left\langle E X^{x_{0}}(t),\left(\begin{array}{c}
0 \\
e_{n}
\end{array}\right)\right\rangle_{V}=\left\langle E_{3} X_{1}^{x_{0}}(t)+E_{4} X_{2}^{x_{0}}(t), e_{n}\right\rangle_{L^{2}(D)} .
$$


Next we have

$$
\begin{aligned}
\left\langle E_{3} X_{1}^{x_{0}}(t), e_{n}\right\rangle_{L^{2}(D)}= & \sum_{k=1}^{\infty} \sum_{l=k+1}^{\infty} \frac{z_{2, k} z_{2, l}}{D_{k, l}}\left\langle E_{k, l, 3} X_{1}^{x_{0}}(t), e_{n}\right\rangle_{L^{2}(D)} \\
= & \sum_{k=1}^{\infty} \sum_{l=k+1}^{\infty} \frac{z_{2, k} z_{2, l}}{D_{k, l}}\left(4 a b \alpha_{l}\left(\alpha_{k}-\alpha_{l}\right)\left\langle X_{1}^{x_{0}}(t), e_{l}\right\rangle_{L^{2}(D)} \delta_{k, n}\right. \\
& \left.+4 a b \alpha_{k}\left(\alpha_{l}-\alpha_{k}\right)\left\langle X_{1}^{x_{0}}(t), e_{k}\right\rangle_{L^{2}(D)} \delta_{n, l}\right) \\
= & \sum_{k=1}^{\infty} \sum_{l=k+1}^{\infty} \frac{z_{2, k} z_{2, l}}{D_{k, l}} 4 a b \alpha_{l}\left(\alpha_{k}-\alpha_{l}\right)\left\langle X_{1}^{x_{0}}(t), e_{l}\right\rangle_{L^{2}(D)} \delta_{k, n} \\
& +\sum_{k=1}^{\infty} \sum_{l=k+1}^{\infty} \frac{z_{2, k} z_{2, l}}{D_{k, l}} 4 a b \alpha_{k}\left(\alpha_{l}-\alpha_{k}\right)\left\langle X_{1}^{x_{0}}(t), e_{k}\right\rangle_{L^{2}(D)} \delta_{n, l} \\
= & (I)+(I I),
\end{aligned}
$$

where we have used the fact that $E_{k, 3}=0$. Furthermore, compute

$$
\begin{aligned}
&\left\langle E_{4} X_{2}^{x_{0}}(t), e_{n}\right\rangle_{L^{2}(D)}= \\
&=\left\langle\sum_{k=1}^{\infty} z_{2, k}^{2} E_{k, 4} X_{2}^{x_{0}}(t)+\sum_{k=1}^{\infty} \sum_{l=k+1}^{\infty} \frac{z_{2, k} z_{2, l}}{D_{k, l}} E_{k, l, 4} X_{2}^{x_{0}}(t), e_{n}\right\rangle_{L^{2}(D)} \\
&=\sum_{k=1}^{\infty} z_{2, k}^{2}\left\langle X_{2}^{x_{0}}(t), e_{k}\right\rangle_{L^{2}(D)} \delta_{k, n} \\
&+\sum_{k=1}^{\infty} \sum_{l=k+1}^{\infty} \frac{z_{2, k} z_{2, l}}{D_{k, l}} 8 a^{2}\left(\alpha_{k}+\alpha_{l}\right)\left\langle X_{2}^{x_{0}}(t), e_{k}\right\rangle_{L^{2}(D)} \delta_{n, l} \\
&+\sum_{k=1}^{\infty} \sum_{l=k+1}^{\infty} \frac{z_{2, k} z_{2, l}}{D_{k, l}} 8 a^{2}\left(\alpha_{k}+\alpha_{l}\right)\left\langle X_{2}^{x_{0}}(t), e_{l}\right\rangle_{L^{2}(D)} \delta_{k, n} \\
&=(I I I)+(I V)+(V) .
\end{aligned}
$$

By the central limit theorem for martingales (see e.g. 7, Proposition 1.22.), Law $(w(T))$ converges weakly to a Gaussian distribution with a zero mean and variance given by the $\mathbb{P}-$ a.s. limit

$$
\lim _{T \rightarrow \infty} \frac{1}{T} \int_{0}^{T} \sum_{n=1}^{\infty} \lambda_{n}((I)+\ldots+(V))^{2} d t
$$

Therefore all the limits in the rest of the proof are considered in the $\mathbb{P}$ - a.s. sense. 
The limits of the cross terms are zero by Lemma 4.4. For example

$$
\begin{aligned}
& \lim _{T \rightarrow \infty} \frac{1}{T} \int_{0}^{T} \sum_{n=1}^{\infty} \lambda_{n}(I)(I I) d t= \\
&=\lim _{T \rightarrow \infty} \frac{1}{T} \int_{0}^{T} \sum_{n=1}^{\infty} \lambda_{n}\left(\sum_{l=n+1}^{\infty} \frac{z_{2, n} z_{2, l}}{D_{n, l}} 4 a b \alpha_{l}\left(\alpha_{n}-\alpha_{l}\right)\left\langle X_{1}^{x_{0}}(t), e_{l}\right\rangle_{L^{2}(D)}\right) \times \\
&\left(\sum_{k=1}^{n-1} \frac{z_{2, k} z_{2, n}}{D_{k, n}} 4 a b \alpha_{k}\left(\alpha_{n}-\alpha_{k}\right)\left\langle X_{1}^{x_{0}}(t), e_{k}\right\rangle_{L^{2}(D)}\right) d t \\
&= \sum_{n=1}^{\infty} \sum_{l=n+1}^{\infty} \sum_{k=1}^{n-1} \lambda_{n} \frac{z_{2, n}^{2} z_{2, l} z_{2, k}}{D_{n, l} D_{k, n}} 16 a^{2} b^{2} \alpha_{l} \alpha_{k}\left(\alpha_{n}-\alpha_{l}\right)\left(\alpha_{n}-\alpha_{k}\right) \times \\
&\left(\lim _{T \rightarrow \infty} \frac{1}{T} \int_{0}^{T}\left\langle X_{1}^{x_{0}}(t), e_{l}\right\rangle_{L^{2}(D)}\left\langle X_{1}^{x_{0}}(t), e_{k}\right\rangle_{L^{2}(D)} d t\right)=0,
\end{aligned}
$$

because $k \neq l,\left\langle X_{1}^{x_{0}}(t), e_{k}\right\rangle_{L^{2}(D)}=\frac{1}{\sqrt{\alpha_{k}}}\left\langle X_{1}^{x_{0}}(t), f_{k}\right\rangle_{\operatorname{Dom}\left((-A)^{\frac{1}{2}}\right)}$ (and similarly with the index $l$ ) and we may use Lemma 4.4, 2).

Also

$$
\begin{aligned}
& \lim _{T \rightarrow \infty} \frac{1}{T} \int_{0}^{T} \sum_{n=1}^{\infty} \lambda_{n}(I)(I I I) d t= \\
&=\lim _{T \rightarrow \infty} \frac{1}{T} \int_{0}^{T} \sum_{n=1}^{\infty} \lambda_{n}\left(\sum_{l=n+1}^{\infty} \frac{z_{2, n} z_{2, l}}{D_{n, l}} 4 a b \alpha_{l}\left(\alpha_{n}-\alpha_{l}\right)\left\langle X_{1}^{x_{0}}(t), e_{l}\right\rangle_{L^{2}(D)}\right) \times \\
&\left(z_{2, n}^{2}\left\langle X_{2}^{x_{0}}(t), e_{n}\right\rangle_{L^{2}(D)}\right) d t \\
&= \sum_{n=1}^{\infty} \sum_{l=n+1}^{\infty} \lambda_{n} \frac{z_{2, n}^{3} z_{2, l}}{D_{n, l}} 4 a b \alpha_{l}\left(\alpha_{n}-\alpha_{l}\right) \times \\
&\left(\lim _{T \rightarrow \infty} \frac{1}{T} \int_{0}^{T}\left\langle X_{1}^{x_{0}}(t), e_{l}\right\rangle_{L^{2}(D)}\left\langle X_{2}^{x_{0}}(t), e_{n}\right\rangle_{L^{2}(D)} d t\right)=0,
\end{aligned}
$$

since $n \neq l$ and the limit follows from Lemma 4.4. 1). The remaining limits of the cross terms are handled similarly.

Now we compute the limits of the "diagonal" terms.

$$
\begin{aligned}
(A) & =\lim _{T \rightarrow \infty} \frac{1}{T} \int_{0}^{T} \sum_{n=1}^{\infty} \lambda_{n}(I)^{2} d t \\
& =\lim _{T \rightarrow \infty} \frac{1}{T} \int_{0}^{T} \sum_{n=1}^{\infty} \lambda_{n}\left(\sum_{l=n+1}^{\infty} \frac{z_{2, n} z_{2, l}}{D_{n, l}} 4 a b \alpha_{l}\left(\alpha_{n}-\alpha_{l}\right)\left\langle X_{1}^{x_{0}}(t), e_{l}\right\rangle_{L^{2}(D)}\right)^{2} d t \\
& \stackrel{(*)}{=} \lim _{T \rightarrow \infty} \frac{1}{T} \int_{0}^{T} \sum_{n=1}^{\infty} \sum_{l=n+1}^{\infty} \lambda_{n} \frac{z_{2, n}^{2} z_{2, l}^{2}}{D_{n, l}^{2}} 16 a^{2} b^{2} \alpha_{l}^{2}\left(\alpha_{n}-\alpha_{l}\right)^{2}\left\langle X_{1}^{x_{0}}(t), e_{l}\right\rangle_{L^{2}(D)}^{2} d t \\
& =\sum_{n=1}^{\infty} \sum_{l=n+1}^{\infty} \frac{1}{D_{n, l}^{2}} 4 a b \lambda_{n} \lambda_{l} \alpha_{l}\left(\alpha_{n}-\alpha_{l}\right)^{2} z_{2, n}^{2} z_{2, l}^{2},
\end{aligned}
$$


since $\lim _{T \rightarrow \infty} \frac{1}{T} \int_{0}^{T}\left\langle X_{1}^{x_{0}}(t), e_{l}\right\rangle_{L^{2}(D)}^{2} d t=\frac{\lambda_{l}}{4 a b \alpha_{l}}$ and in the equality $(*)$ we have also used Lemma 4.4 for the cross summands.

In a similar manner, we have

$$
\begin{aligned}
(B) & =\lim _{T \rightarrow \infty} \frac{1}{T} \int_{0}^{T} \sum_{n=1}^{\infty} \lambda_{n}(I I)^{2} d t \\
& =\lim _{T \rightarrow \infty} \frac{1}{T} \int_{0}^{T} \sum_{n=1}^{\infty} \lambda_{n}\left(\sum_{k=1}^{n-1} \frac{z_{2, k} z_{2, n}}{D_{k, n}} 4 a b \alpha_{k}\left(\alpha_{n}-\alpha_{k}\right)\left\langle X_{1}^{x_{0}}(t), e_{k}\right\rangle_{L^{2}(D)}\right)^{2} d t \\
& \stackrel{(*)}{=} \lim _{T \rightarrow \infty} \frac{1}{T} \int_{0}^{T} \sum_{n=1}^{\infty} \sum_{k=1}^{n-1} \lambda_{n} \frac{z_{2, k}^{2} z_{2, n}^{2}}{D_{k, n}^{2}} 16 a^{2} b^{2} \alpha_{k}^{2}\left(\alpha_{n}-\alpha_{k}\right)^{2}\left\langle X_{1}^{x_{0}}(t), e_{k}\right\rangle_{L^{2}(D)}^{2} d t \\
& =\sum_{n=1}^{\infty} \sum_{k=1}^{n-1} \frac{1}{D_{k, n}^{2}} 4 a b \lambda_{n} \lambda_{k} \alpha_{k}\left(\alpha_{n}-\alpha_{k}\right)^{2} z_{2, k}^{2} z_{2, n}^{2},
\end{aligned}
$$

$$
\begin{aligned}
(C) & =\lim _{T \rightarrow \infty} \frac{1}{T} \int_{0}^{T} \sum_{n=1}^{\infty} \lambda_{n}(I I I)^{2} d t \\
& =\lim _{T \rightarrow \infty} \frac{1}{T} \int_{0}^{T} \sum_{n=1}^{\infty} \lambda_{n} z_{2, n}^{4}\left\langle X_{2}^{x_{0}}(t), e_{n}\right\rangle_{L^{2}(D)}^{2} d t \\
& =\frac{1}{4 a} \sum_{n=1}^{\infty} \lambda_{n}^{2} z_{2, n}^{4},
\end{aligned}
$$

since $\lim _{T \rightarrow \infty} \frac{1}{T} \int_{0}^{T}\left\langle X_{2}^{x_{0}}(t), e_{n}\right\rangle_{L^{2}(D)}^{2} d t=\frac{\lambda_{n}}{4 a}$.

Next, we have

$$
\begin{aligned}
(D) & =\lim _{T \rightarrow \infty} \frac{1}{T} \int_{0}^{T} \sum_{n=1}^{\infty} \lambda_{n}(I V)^{2} d t \\
& =\lim _{T \rightarrow \infty} \frac{1}{T} \int_{0}^{T} \sum_{n=1}^{\infty} \lambda_{n}\left(\sum_{k=1}^{n-1} \frac{z_{2, k} z_{2, n}}{D_{k, n}} 8 a^{2}\left(\alpha_{k}+\alpha_{n}\right)\left\langle X_{2}^{x_{0}}(t), e_{k}\right\rangle_{L^{2}(D)}\right)^{2} d t \\
& \stackrel{(*)}{=} \lim _{T \rightarrow \infty} \frac{1}{T} \int_{0}^{T} \sum_{n=1}^{\infty} \sum_{k=1}^{n-1} \lambda_{n} \frac{z_{2, k}^{2} z_{2, n}^{2}}{D_{k, n}^{2}} 64 a^{4}\left(\alpha_{k}+\alpha_{n}\right)^{2}\left\langle X_{2}^{x_{0}}(t), e_{k}\right\rangle_{L^{2}(D)}^{2} d t \\
& =\sum_{n=1}^{\infty} \sum_{k=1}^{n-1} \frac{1}{D_{k, n}^{2}} 16 a^{3} \lambda_{n} \lambda_{k}\left(\alpha_{k}+\alpha_{n}\right)^{2} z_{2, k}^{2} z_{2, n}^{2},
\end{aligned}
$$




$$
\begin{aligned}
(E) & =\lim _{T \rightarrow \infty} \frac{1}{T} \int_{0}^{T} \sum_{n=1}^{\infty} \lambda_{n}(V)^{2} d t \\
& =\lim _{T \rightarrow \infty} \frac{1}{T} \int_{0}^{T} \sum_{n=1}^{\infty} \lambda_{n}\left(\sum_{l=n+1}^{\infty} \frac{z_{2, n} z_{2, l}}{D_{n, l}} 8 a^{2}\left(\alpha_{n}+\alpha_{l}\right)\left\langle X_{2}^{x_{0}}(t), e_{l}\right\rangle_{L^{2}(D)}\right)^{2} d t \\
& \stackrel{(*)}{=} \lim _{T \rightarrow \infty} \frac{1}{T} \int_{0}^{T} \sum_{n=1}^{\infty} \sum_{l=n+1}^{\infty} \lambda_{n} \frac{z_{2, n}^{2} z_{2, l}^{2}}{D_{n, l}^{2}} 64 a^{4}\left(\alpha_{n}+\alpha_{l}\right)^{2}\left\langle X_{2}^{x_{0}}(t), e_{l}\right\rangle_{L^{2}(D)}^{2} d t \\
& =\sum_{n=1}^{\infty} \sum_{l=n+1}^{\infty} \frac{1}{D_{n, l}^{2}} 16 a^{3} \lambda_{n} \lambda_{l}\left(\alpha_{n}+\alpha_{l}\right)^{2} z_{2, n}^{2} z_{2, l}^{2} .
\end{aligned}
$$

The resulting formula for the limiting variance of $w(T)$ is the sum of the five above terms, however it may be further simplified. Since

$$
\sum_{n=1}^{\infty} \sum_{k=1}^{n-1} a_{n, k}=\sum_{k=1}^{\infty} \sum_{n=k+1}^{\infty} a_{n, k}, \quad \forall a_{n, k} \in \mathbb{R},
$$

we may switch the sums in the term $(B)$ and by changing indices $n \mapsto k, l \mapsto n$ in the term $(A)$, we arrive at

$$
(A)+(B)=\sum_{k=1}^{\infty} \sum_{n=k+1}^{\infty} \frac{1}{D_{k, n}^{2}} 4 a b \lambda_{k} \lambda_{n}\left(\alpha_{k}+\alpha_{n}\right)\left(\alpha_{n}-\alpha_{k}\right)^{2} z_{2, k}^{2} z_{2, n}^{2} .
$$

Similary, if we switch the sums in the term $(D)$ and change indices $n \mapsto k, l \mapsto n$ in the term $(E)$, we find out that $(D)=(E)$, so

$$
(D)+(E)=\sum_{k=1}^{\infty} \sum_{n=k+1}^{\infty} \frac{1}{D_{k, n}^{2}} 32 a^{3} \lambda_{k} \lambda_{n}\left(\alpha_{k}+\alpha_{n}\right)^{2} z_{2, k}^{2} z_{2, n}^{2}
$$

and consequently

$$
\begin{aligned}
& (A)+(B)+(D)+(E)= \\
= & \sum_{k=1}^{\infty} \sum_{n=k+1}^{\infty} \frac{1}{D_{k, n}^{2}} 4 a \lambda_{k} \lambda_{n}\left(\alpha_{k}+\alpha_{n}\right) z_{2, k}^{2} z_{2, n}^{2}\left(b\left(\alpha_{n}-\alpha_{k}\right)^{2}+8 a^{2}\left(\alpha_{k}+\alpha_{n}\right)\right) \\
= & \sum_{k=1}^{\infty} \sum_{n=k+1}^{\infty} \frac{1}{D_{k, n}} 4 a \lambda_{k} \lambda_{n}\left(\alpha_{k}+\alpha_{n}\right) z_{2, k}^{2} z_{2, n}^{2}
\end{aligned}
$$

Since

$$
\sum_{k=1}^{\infty} \sum_{n=k+1}^{\infty} a_{n, k}=\frac{1}{2} \sum_{k=1}^{\infty} \sum_{n=1, n \neq k}^{\infty} a_{n, k}, \quad \text { if } \forall k \in \mathbb{N} \forall n \in \mathbb{N} \quad a_{k, n}=a_{n, k},
$$

the sum on the right-hand side of (4.9) equals to

$$
2 a \sum_{k=1}^{\infty} \sum_{n=1, n \neq k}^{\infty} \frac{1}{D_{k, n}} \lambda_{k} \lambda_{n}\left(\alpha_{k}+\alpha_{n}\right) z_{2, k}^{2} z_{2, n}^{2} .
$$

The summand $(C)$ is the corresponding sum to (4.10), where $n=k$, so we may add it and we end up with the formula for the limiting variance of $w(T)$, that is

$$
\operatorname{Var}(w(T))=2 a \sum_{k=1}^{\infty} \sum_{n=1}^{\infty} \frac{1}{D_{k, n}} \lambda_{k} \lambda_{n}\left(\alpha_{k}+\alpha_{n}\right) z_{2, k}^{2} z_{2, n}^{2}, \quad T \rightarrow \infty .
$$


Since the multiplicative factor $-\frac{1}{\frac{2}{T} \int_{0}^{T}\left\langle X_{2}^{x_{0}}(t), z_{2}\right\rangle_{L^{2}(D)}^{2} d t}$ of $w(T)$ on the right-hand side of (4.8) converges to $\frac{-2 a}{\left\langle Q z_{2}, z_{2}\right\rangle_{L^{2}(D)}}$ as $T \rightarrow \infty$, we arrive at

$$
\begin{aligned}
& \operatorname{Law}\left(\sqrt{T}\left(\bar{a}_{T, z_{2}}-a\right)\right) \stackrel{w^{*}}{\longrightarrow} \\
& \quad N\left(0, \frac{8 a^{3}}{\left\langle Q z_{2}, z_{2}\right\rangle_{L^{2}(D)}^{2}} \sum_{k=1}^{\infty} \sum_{n=1}^{\infty} \frac{\lambda_{k} \lambda_{n}\left(\alpha_{k}+\alpha_{n}\right) z_{2, k}^{2} z_{2, n}^{2}}{b\left(\alpha_{k}-\alpha_{n}\right)^{2}+8 a^{2}\left(\alpha_{k}+\alpha_{n}\right)}\right), \quad T \rightarrow \infty .
\end{aligned}
$$

4.2. Asymptotic normality of the estimator $\bar{b}_{T, z_{1}, z_{2}}$. The estimator $\bar{b}_{T, z_{1}, z_{2}}$ (defined by (3.5) ) is also asymptotically normal. The proof uses similiar technique as the proof of Theorem 4.6, so the setup and auxiliary Lemmas will be analogous to those in previous subsection.

Let $k \in \mathbb{N}$ be arbitrary and define the operator $F_{k}: V \rightarrow V$ by

$$
F_{k} x=F_{k}\left(\begin{array}{c}
x_{1} \\
x_{2}
\end{array}\right)=\left(\begin{array}{ll}
F_{k, 1} & F_{k, 2} \\
F_{k, 3} & F_{k, 4}
\end{array}\right)\left(\begin{array}{c}
x_{1} \\
x_{2}
\end{array}\right), \quad \forall x=\left(\begin{array}{c}
x_{1} \\
x_{2}
\end{array}\right) \in V
$$

where

$$
\begin{aligned}
& F_{k, 1}: x_{1} \longmapsto\left(b+\frac{4 a^{2}}{\alpha_{k}}\right)\left\langle x_{1}, e_{k}\right\rangle_{L^{2}(D)} e_{k}, \\
& F_{k, 2}: x_{2} \longmapsto \frac{2 a}{\alpha_{k}}\left\langle x_{2}, e_{k}\right\rangle_{L^{2}(D)} e_{k}, \\
& F_{k, 3}: x_{1} \longmapsto 2 a\left\langle x_{1}, e_{k}\right\rangle_{L^{2}(D)} e_{k}, \\
& F_{k, 4}: x_{2} \longmapsto\left\langle x_{2}, e_{k}\right\rangle_{L^{2}(D)} e_{k},
\end{aligned}
$$

for any $x_{1} \in \operatorname{Dom}\left((-A)^{\frac{1}{2}}\right)$ and $x_{2} \in L^{2}(D)$.

Let $k, l \in \mathbb{N}$ be arbitrary and define the operator $F_{k, l}: V \rightarrow V$ by

$$
F_{k, l} x=F_{k}\left(\begin{array}{c}
x_{1} \\
x_{2}
\end{array}\right)=\frac{1}{D_{k, l}}\left(\begin{array}{cc}
F_{k, l, 1} & F_{k, l, 2} \\
F_{k, l, 3} & F_{k, l, 4}
\end{array}\right)\left(\begin{array}{c}
x_{1} \\
x_{2}
\end{array}\right), \quad \forall x=\left(\begin{array}{c}
x_{1} \\
x_{2}
\end{array}\right) \in V,
$$

where

$$
\begin{aligned}
F_{k, l, 1}: x_{1} \longmapsto & 8 a^{2} \sqrt{\frac{\alpha_{l}}{\alpha_{k}}}\left(8 a^{2}+b\left(\alpha_{k}+\alpha_{l}\right)\right)\left\langle x_{1}, e_{l}\right\rangle_{L^{2}(D)} e_{k} \\
& +8 a^{2} \sqrt{\frac{\alpha_{k}}{\alpha_{l}}}\left(8 a^{2}+b\left(\alpha_{k}+\alpha_{l}\right)\right)\left\langle x_{1}, e_{k}\right\rangle_{L^{2}(D)} e_{l}, \\
F_{k, l, 2}: x_{2} \longmapsto & 4 a \sqrt{\frac{\alpha_{k}}{\alpha_{l}}}\left(8 a^{2}+b\left(\alpha_{k}-\alpha_{l}\right)\right)\left\langle x_{2}, e_{k}\right\rangle_{L^{2}(D)} e_{l} \\
+ & 4 a \sqrt{\frac{\alpha_{l}}{\alpha_{k}}}\left(8 a^{2}+b\left(\alpha_{l}-\alpha_{k}\right)\right)\left\langle x_{2}, e_{l}\right\rangle_{L^{2}(D)} e_{k}, \\
F_{k, l, 3}: x_{1} \longmapsto & 4 a \sqrt{\alpha_{k} \alpha_{l}}\left(8 a^{2}+b\left(\alpha_{k}-\alpha_{l}\right)\right)\left\langle x_{1}, e_{l}\right\rangle_{L^{2}(D)} e_{k} \\
& +4 a \sqrt{\alpha_{k} \alpha_{l}}\left(8 a^{2}+b\left(\alpha_{l}-\alpha_{k}\right)\right)\left\langle x_{1}, e_{k}\right\rangle_{L^{2}(D)} e_{l}, \\
F_{k, l, 4}: x_{2} \longmapsto & 16 a^{2} \sqrt{\alpha_{k} \alpha_{l}}\left\langle x_{2}, e_{k}\right\rangle_{L^{2}(D)} e_{l}+16 a^{2} \sqrt{\alpha_{k} \alpha_{l}}\left\langle x_{2}, e_{l}\right\rangle_{L^{2}(D)} e_{k},
\end{aligned}
$$

for any $x_{1} \in \operatorname{Dom}\left((-A)^{\frac{1}{2}}\right)$ and $x_{2} \in L^{2}(D)$ with $D_{k, l}$ defined above.

The properties of the operators $F_{k}$ and $F_{k, l}$ needed in the sequel are summarized in the following Lemma. 
Lemma 4.7. 1) The operator $F_{k} \in \mathcal{L}(V)$ is self-adjoint for any given $k \in \mathbb{N}$. Moreover,

$$
\left\langle F_{k} x, \mathcal{A} x\right\rangle_{V}=-2 a b\left\langle x_{1}, f_{k}\right\rangle_{\operatorname{Dom}\left((-A)^{\frac{1}{2}}\right)}^{2},
$$

for any $x=\left(x_{1}, x_{2}\right)^{\top} \in \operatorname{Dom}(\mathcal{A})$.

2) The operator $F_{k, l} \in \mathcal{L}(V)$ is self-adjoint for any given $k, l \in \mathbb{N}$. Moreover,

$$
\left\langle F_{k, l} x, \mathcal{A} x\right\rangle_{V}=-4 a b\left\langle x_{1}, f_{k}\right\rangle_{\operatorname{Dom}\left((-A)^{\frac{1}{2}}\right)}\left\langle x_{1}, f_{l}\right\rangle_{\operatorname{Dom}\left((-A)^{\frac{1}{2}}\right)},
$$

for any $x=\left(x_{1}, x_{2}\right)^{\top} \in \operatorname{Dom}(\mathcal{A})$.

Proof. 1) Let $k \in \mathbb{N}$ be arbitrary. Obviously $F_{k} \in \mathcal{L}(V)$ and for $x=\left(x_{1}, x_{2}\right)^{\top} \in V$ and $y=\left(y_{1}, y_{2}\right)^{\top} \in V$ we have

$$
\begin{aligned}
\left\langle F_{k} x, y\right\rangle_{V}= & \left\langle\left(\begin{array}{c}
F_{k, 1} x_{1}+F_{k, 2} x_{2} \\
F_{k, 3} x_{1}+F_{k, 4} x_{2}
\end{array}\right),\left(\begin{array}{c}
y_{1} \\
y_{2}
\end{array}\right)\right\rangle_{V} \\
= & \left(b+\frac{4 a^{2}}{\alpha_{k}}\right)\left\langle x_{1}, e_{k}\right\rangle_{L^{2}(D)}\left\langle y_{1}, e_{k}\right\rangle_{\operatorname{Dom}\left((-A)^{\frac{1}{2}}\right)} \\
& +\frac{2 a}{\alpha_{k}}\left\langle x_{2}, e_{k}\right\rangle_{L^{2}(D)}\left\langle y_{1}, e_{k}\right\rangle_{\operatorname{Dom}\left((-A)^{\frac{1}{2}}\right)} \\
& +2 a\left\langle x_{1}, e_{k}\right\rangle_{L^{2}(D)}\left\langle y_{2}, e_{k}\right\rangle_{L^{2}(D)}+\left\langle x_{2}, e_{k}\right\rangle_{L^{2}(D)}\left\langle y_{2}, e_{k}\right\rangle_{L^{2}(D)} \\
= & \left(b \alpha_{k}+4 a^{2}\right)\left\langle x_{1}, e_{k}\right\rangle_{L^{2}(D)}\left\langle y_{1}, e_{k}\right\rangle_{L^{2}(D)}+2 a\left\langle x_{2}, e_{k}\right\rangle_{L^{2}(D)}\left\langle y_{1}, e_{k}\right\rangle_{L^{2}(D)} \\
& +2 a\left\langle x_{1}, e_{k}\right\rangle_{L^{2}(D)}\left\langle y_{2}, e_{k}\right\rangle_{L^{2}(D)}+\left\langle x_{2}, e_{k}\right\rangle_{L^{2}(D)}\left\langle y_{2}, e_{k}\right\rangle_{L^{2}(D)} \\
= & \left\langle x, F_{k} y\right\rangle_{V},
\end{aligned}
$$

hence $F_{k}=F_{k}^{*}$. Moreover, for every $x=\left(x_{1}, x_{2}\right)^{\top} \in \operatorname{Dom}(\mathcal{A})$ we have

$$
\begin{aligned}
\left\langle F_{k} x, \mathcal{A} x\right\rangle_{V}= & \left\langle\left(\begin{array}{c}
F_{k, 1} x_{1}+F_{k, 2} x_{2} \\
F_{k, 3} x_{1}+F_{k, 4} x_{2}
\end{array}\right),\left(\begin{array}{c}
x_{2} \\
b A x_{1}-2 a x_{2}
\end{array}\right)\right\rangle_{V} \\
= & \left(b+\frac{4 a^{2}}{\alpha_{k}}\right)\left\langle x_{1}, e_{k}\right\rangle_{L^{2}(D)}\left\langle(-A)^{\frac{1}{2}} x_{2},(-A)^{\frac{1}{2}} e_{k}\right\rangle_{L^{2}(D)} \\
& +\frac{2 a}{\alpha_{k}}\left\langle x_{2}, e_{k}\right\rangle_{L^{2}(D)}\left\langle(-A)^{\frac{1}{2}} x_{2},(-A)^{\frac{1}{2}} e_{k}\right\rangle_{L^{2}(D)} \\
& +2 a b\left\langle x_{1}, e_{k}\right\rangle_{L^{2}(D)}\left\langle A x_{1}, e_{k}\right\rangle_{L^{2}(D)}-4 a^{2}\left\langle x_{1}, e_{k}\right\rangle_{L^{2}(D)}\left\langle x_{2}, e_{k}\right\rangle_{L^{2}(D)} \\
& +b\left\langle x_{2}, e_{k}\right\rangle_{L^{2}(D)}\left\langle A x_{1}, e_{k}\right\rangle_{L^{2}(D)}-2 a\left\langle x_{2}, e_{k}\right\rangle_{L^{2}(D)}^{2} \\
= & 2 a b\left\langle x_{1}, e_{k}\right\rangle_{L^{2}(D)}\left\langle A x_{1}, e_{k}\right\rangle_{L^{2}(D)} \\
= & -2 a b\left\langle x_{1}, f_{k}\right\rangle_{\operatorname{Dom}\left((-A)^{\frac{1}{2}}\right)}^{2}
\end{aligned}
$$

2) Let $k, l \in \mathbb{N}$ be arbitrary. It is clear that $F_{k, l} \in \mathcal{L}(V)$ and similarly as above it is possible to verify that $F_{k, l}=F_{k, l}^{*}$ and that (4.12) holds true for any $x \in \operatorname{Dom}(\mathcal{A})$.

Choose $0 \neq z_{1} \in \operatorname{Dom}\left((-A)^{\frac{1}{2}}\right)$ taking the form

$$
z_{1}=\sum_{k=1}^{\infty}\left\langle z_{1}, f_{k}\right\rangle_{\operatorname{Dom}\left((-A)^{\frac{1}{2}}\right)} f_{k}=\sum_{k=1}^{\infty} z_{1, k} f_{k}
$$


that is, $\left\{z_{1, k}, k \in \mathbb{N}\right\}$ is the set of coordinates of the element $z_{1}$ with respect to the orthonormal basis in $\operatorname{Dom}\left((-A)^{\frac{1}{2}}\right)$. Finally, define the operator $F: V \rightarrow V$ by

$$
F=\sum_{k=1}^{\infty} z_{1, k}^{2} F_{k}+\sum_{k=1}^{\infty} \sum_{l=k+1}^{\infty} z_{1, k} z_{1, l} F_{k, l} .
$$

The properties of the operator $F$ are summarized in the following Lemma.

Lemma 4.8. The operator $F \in \mathcal{L}(V)$. Moreover, it is self-adjoint and

$$
\langle F x, \mathcal{A} x\rangle_{V}=-2 a b\left\langle x_{1}, z_{1}\right\rangle_{\operatorname{Dom}\left((-A)^{\frac{1}{2}}\right)}^{2}, \quad \forall x=\left(\begin{array}{c}
x_{1} \\
x_{2}
\end{array}\right) \in \operatorname{Dom}(\mathcal{A}) .
$$

Proof. Using inequalities in the proof of Lemma 4.2 it is possible to verify that $F \in \mathcal{L}(V)$. Moreover, the linear combination of the self-adjoint operators is also the self-adjoint operator, hence $F=F^{*}$.

The property (4.13) comes from (4.11) and (4.12). The proof is analogous to the proof of Lemma 4.2

We will also need an alternative representation for the process $\frac{1}{T} \int_{0}^{T}\left\langle X_{1}^{x_{0}}(t), z_{1}\right\rangle_{\operatorname{Dom}\left((-A)^{\frac{1}{2}}\right)}^{2} d t$.

Lemma 4.9. The process $\frac{1}{T} \int_{0}^{T}\left\langle X_{1}^{x_{0}}(t), z_{1}\right\rangle_{\operatorname{Dom}\left((-A)^{\frac{1}{2}}\right)}^{2} d t$ admits the following representation

$$
\begin{aligned}
\frac{1}{T} \int_{0}^{T}\left\langle X_{1}^{x_{0}}(t), z_{1}\right\rangle_{\operatorname{Dom}\left((-A)^{\frac{1}{2}}\right) d t=}^{2} & -\frac{1}{4 a b T}\left(\left\langle F X^{x_{0}}(T), X^{x_{0}}(T)\right\rangle_{V}-\left\langle F x_{0}, x_{0}\right\rangle_{V}\right) \\
& +\frac{1}{2 a b T} \int_{0}^{T}\left\langle F X^{x_{0}}(t), \Phi d B(t)\right\rangle_{V} \\
& +\frac{1}{4 a b}\left\langle Q z_{1}, z_{1}\right\rangle_{\operatorname{Dom}\left((-A)^{\frac{1}{2}}\right)} .
\end{aligned}
$$

Proof. Define the function $g_{1}: V \rightarrow \mathbb{R}$ by

$$
g_{1}(x)=\langle F x, x\rangle_{V}, \quad \forall x \in V .
$$

The application of Itô's formula to the function $g_{1}\left(X^{x_{0}, N}(t)\right)$ (we also have to use suitable projections, see the proof of Lemma 4.3), yields

$$
d g_{1}\left(X^{x_{0}, N}(t)\right)=2\left\langle F X^{x_{0}, N}(t), d X^{x_{0}, N}(t)\right\rangle_{V}+\frac{1}{2} \operatorname{Tr}\left(2 F \Phi \Phi^{*}\right) d t .
$$

We also start with simplification of the second term

$$
\begin{aligned}
\operatorname{Tr}\left(F \Phi \Phi^{*}\right) & =\operatorname{Tr}\left(F_{4} Q\right) \\
& =\sum_{k=1}^{\infty} z_{1, k}^{2} \operatorname{Tr}\left(F_{k, 4} Q\right)+\sum_{k=1}^{\infty} \sum_{l=k+1}^{\infty} \frac{z_{1, k} z_{1, l}}{D_{k, l}} \operatorname{Tr}\left(F_{k, l, 4} Q\right) \\
& =\left\langle Q z_{1}, z_{1}\right\rangle_{\operatorname{Dom}\left((-A)^{\frac{1}{2}}\right)}
\end{aligned}
$$

since $\operatorname{Tr}\left(F_{k, 4} Q\right)=\lambda_{k}, \operatorname{Tr}\left(F_{k, l, 4} Q\right)=0$, because $k \neq l$, and

$$
\sum_{k=1}^{\infty} \lambda_{k} z_{1, k}^{2}=\left\langle Q z_{1}, z_{1}\right\rangle_{\operatorname{Dom}\left((-A)^{\frac{1}{2}}\right)} .
$$


Lemma 4.8 and the formula (4.15) imply

$$
\begin{aligned}
d g_{1}\left(X^{x_{0}, N}(t)\right)= & 2\left\langle F X^{x_{0}, N}(t), \mathcal{A} X^{x_{0}, N}(t)\right\rangle_{V} d t+2\left\langle F X^{x_{0}, N}(t), \Phi d B(t)\right\rangle_{V} \\
& +\left\langle Q z_{1}, z_{1}\right\rangle_{\operatorname{Dom}\left((-A)^{\frac{1}{2}}\right)} d t \\
= & -4 a b\left\langle X_{1}^{x_{0}, N}(t), z_{1}\right\rangle_{\operatorname{Dom}\left((-A)^{\frac{1}{2}}\right)}^{2} d t+2\left\langle F X^{x_{0}, N}(t), \Phi d B(t)\right\rangle_{V} \\
& +\left\langle Q z_{1}, z_{1}\right\rangle_{\operatorname{Dom}\left((-A)^{\frac{1}{2}}\right)} d t .
\end{aligned}
$$

By integrating the above formula over the interval $(0, T)$ and passing $N$ to infinity, we arrive at (4.14).

Denote

$$
Q_{1}:=\left\langle Q z_{1}, z_{1}\right\rangle_{\operatorname{Dom}\left((-A)^{\frac{1}{2}}\right)}, \quad Q_{2}:=\left\langle Q z_{2}, z_{2}\right\rangle_{L^{2}(D)} .
$$

Asymptotic normality of the estimator $\bar{b}_{T, z_{1}, z_{2}}$ is formulated in the following Theorem.

Theorem 4.10. Let $0 \neq z_{1} \in \operatorname{Dom}\left((-A)^{\frac{1}{2}}\right), 0 \neq z_{2} \in L^{2}(D)$ be arbitrary. The estimator $\bar{b}_{T, z_{1}, z_{2}}$ is asymptotically normal, i.e., Law $\left(\sqrt{T}\left(\bar{b}_{T, z_{1}, z_{2}}-b\right)\right)$ converges weakly to a centered Gaussian distribution with variance given by

$$
\begin{aligned}
& \frac{64 a^{3} b}{Q_{1}^{2}} \sum_{k=1}^{\infty} \sum_{n=1}^{\infty} \frac{\lambda_{k} \lambda_{n} z_{1, k}^{2} z_{1, n}^{2}}{b\left(\alpha_{k}-\alpha_{n}\right)^{2}+8 a^{2}\left(\alpha_{k}+\alpha_{n}\right)} \\
& +\frac{8 a b^{2}}{Q_{1}^{2} Q_{2}^{2}} \sum_{k=1}^{\infty} \sum_{n=1}^{\infty} \frac{\lambda_{k} \lambda_{n}}{b\left(\alpha_{k}-\alpha_{n}\right)^{2}+8 a^{2}\left(\alpha_{k}+\alpha_{n}\right)}\left(\left(Q_{1} z_{2, k} z_{2, n} \sqrt{\alpha_{k}}-Q_{2} z_{1, k} z_{1, n} \sqrt{\alpha_{n}}\right)^{2}\right. \\
& \left.+\left(Q_{1} z_{2, k} z_{2, n} \sqrt{\alpha_{n}}-Q_{2} z_{1, k} z_{1, n} \sqrt{\alpha_{k}}\right)^{2}\right) .
\end{aligned}
$$


Proof. Set $0 \neq z_{1} \in \operatorname{Dom}\left((-A)^{\frac{1}{2}}\right)$ and $0 \neq z_{2} \in L^{2}(D)$. Using formula (3.5) for the estimator $\bar{b}_{T, z_{1}, z_{2}}$ and Lemmas 4.3 and 4.9 , we obtain

$$
\begin{aligned}
\sqrt{T} & \left(\bar{b}_{T, z_{1}, z_{2}}-b\right)= \\
= & \frac{\sqrt{T}}{Q_{2} \frac{1}{T} \int_{0}^{T}\left\langle X_{1}^{x_{0}}(t), z_{1}\right\rangle_{\operatorname{Dom}\left((-A)^{\frac{1}{2}}\right)}^{2} d t}\left(\frac{Q_{1}}{T} \int_{0}^{T}\left\langle X_{2}^{x_{0}}(t), z_{2}\right\rangle_{L^{2}(D)}^{2} d t\right. \\
& \left.-\frac{b Q_{2}}{T} \int_{0}^{T}\left\langle X_{1}^{x_{0}}(t), z_{1}\right\rangle_{\operatorname{Dom}\left((-A)^{\frac{1}{2}}\right)}^{2} d t\right) \\
= & \frac{\sqrt{T}}{Q_{2} \frac{1}{T} \int_{0}^{T}\left\langle X_{1}^{x_{0}}(t), z_{1}\right\rangle_{\operatorname{Dom}\left((-A)^{\frac{1}{2}}\right)}^{2} d t}\left(-\frac{Q_{1}}{4 a T}\left(\left\langle E X^{x_{0}}(T), X^{x_{0}}(T)\right\rangle_{V}\right.\right. \\
& \left.-\left\langle E x_{0}, x_{0}\right\rangle_{V}\right)+\frac{Q_{1}}{2 a T} \int_{0}^{T}\left\langle E X^{x_{0}}(t), \Phi d B(t)\right\rangle_{V}+ \\
& \left.+\frac{Q_{2}}{4 a T}\left(\left\langle F X^{x_{0}}(T), X^{x_{0}}(T)\right\rangle_{V}-\left\langle F x_{0}, x_{0}\right\rangle_{V}\right)-\frac{Q_{2}}{2 a T} \int_{0}^{T}\left\langle F X^{x_{0}}(t), \Phi d B(t)\right\rangle_{V}\right) \\
= & -\frac{Q_{1}}{4 a Q_{2} \frac{1}{T} \int_{0}^{T}\left\langle X_{1}^{x_{0}}(t), z_{1}\right\rangle_{\operatorname{Dom}\left((-A)^{\frac{1}{2}}\right)}^{2} d t} \frac{1}{\sqrt{T}}\left(\left\langle E X^{x_{0}}(T), X^{x_{0}}(T)\right\rangle_{V}-\left\langle E x_{0}, x_{0}\right\rangle_{V}\right) \\
& +\frac{1}{4 a \frac{1}{T} \int_{0}^{T}\left\langle X_{1}^{x_{0}}(t), z_{1}\right\rangle_{\operatorname{Dom}\left((-A)^{\frac{1}{2}}\right)}^{2} d t} \frac{1}{\sqrt{T}}\left(\left\langle F X^{x_{0}}(T), X^{x_{0}}(T)\right\rangle_{V}-\left\langle F x_{0}, x_{0}\right\rangle_{V}\right) \\
& +\frac{1}{2 a Q_{2} \frac{1}{T} \int_{0}^{T}\left\langle X_{1}^{x_{0}}(t), z_{1}\right\rangle_{\operatorname{Dom}\left((-A)^{\frac{1}{2}}\right)}^{2} d t} \frac{1}{\sqrt{T}} \int_{0}^{T}\left\langle\left(Q_{1} E-Q_{2} F\right) X^{x_{0}}(t), \Phi d B(t)\right\rangle_{V} .
\end{aligned}
$$

The first two terms converge to zero in probability as $T \rightarrow \infty$, since

$$
\lim _{T \rightarrow \infty} \frac{1}{T} \int_{0}^{T}\left\langle X_{1}^{x_{0}}(t), z_{1}\right\rangle_{\operatorname{Dom}\left((-A)^{\frac{1}{2}}\right)}^{2} d t=\frac{Q_{1}}{4 a b}, \quad \mathbb{P}-\text { a.s. }
$$

by Theorem 3.2 and

$$
\begin{aligned}
& \lim _{T \rightarrow \infty} \frac{1}{\sqrt{T}}\left(\left\langle E X^{x_{0}}(T), X^{x_{0}}(T)\right\rangle_{V}-\left\langle E x_{0}, x_{0}\right\rangle_{V}\right)=0, \quad \text { in } L^{1}(\Omega), \\
& \lim _{T \rightarrow \infty} \frac{1}{\sqrt{T}}\left(\left\langle F X^{x_{0}}(T), X^{x_{0}}(T)\right\rangle_{V}-\left\langle F x_{0}, x_{0}\right\rangle_{V}\right)=0, \quad \text { in } L^{1}(\Omega),
\end{aligned}
$$

by Lemma 4.5. Define

$$
\begin{aligned}
w_{1}(T) & =\frac{1}{\sqrt{T}} \int_{0}^{T}\left\langle\left(Q_{1} E-Q_{2} F\right) X^{x_{0}}(t), \Phi d B(t)\right\rangle_{V} \\
& =\frac{1}{\sqrt{T}} \int_{0}^{T} \sum_{n=1}^{\infty} \sqrt{\lambda_{n}}\left\langle\left(Q_{1} E-Q_{2} F\right) X^{x_{0}}(t),\left(\begin{array}{c}
0 \\
e_{n}
\end{array}\right)\right\rangle_{V} d \beta_{n}(t),
\end{aligned}
$$

where we have used the representation of $V$-valued Brownian motion $B(t)$. 
Furthermore, we compute the scalar product in the above series

$$
\begin{aligned}
& \left\langle\left(Q_{1} E-Q_{2} F\right) X^{x_{0}}(t),\left(\begin{array}{c}
0 \\
e_{n}
\end{array}\right)\right\rangle_{V}= \\
& =\left\langle\left(Q_{1} E_{3}-Q_{2} F_{3}\right) X_{1}^{x_{0}}(t), e_{n}\right\rangle_{L^{2}(D)}+\left\langle\left(Q_{1} E_{4}-Q_{2} F_{4}\right) X_{2}^{x_{0}}(t), e_{n}\right\rangle_{L^{2}(D)} .
\end{aligned}
$$

By the definition of the operators $E_{3}, F_{3}, E_{4}, F_{4}$, we have

$$
\begin{aligned}
\langle & \left.\left(Q_{1} E_{3}-Q_{2} F_{3}\right) X_{1}^{x_{0}}(t), e_{n}\right\rangle_{L^{2}(D)}= \\
= & \sum_{k=1}^{\infty} \sum_{l=k+1}^{\infty} \frac{4 a}{D_{k, l}}\left(Q_{1} z_{2, k} z_{2, l} b \alpha_{l}\left(\alpha_{k}-\alpha_{l}\right)-Q_{2} z_{1, k} z_{1, l} \sqrt{\alpha_{k} \alpha_{l}}\left(8 a^{2}+b\left(\alpha_{k}-\alpha_{l}\right)\right)\right) \times \\
& \left\langle X_{1}^{x_{0}}(t), e_{l}\right\rangle_{L^{2}(D)} \delta_{k, n} \\
& +\sum_{k=1}^{\infty} \sum_{l=k+1}^{\infty} \frac{4 a}{D_{k, l}}\left(Q_{1} z_{2, k} z_{2, l} b \alpha_{k}\left(\alpha_{l}-\alpha_{k}\right)-Q_{2} z_{1, k} z_{1, l} \sqrt{\alpha_{k} \alpha_{l}}\left(8 a^{2}+b\left(\alpha_{l}-\alpha_{k}\right)\right)\right) \times \\
& \left\langle X_{1}^{x_{0}}(t), e_{k}\right\rangle_{L^{2}(D)} \delta_{n, l} \\
& -\sum_{k=1}^{\infty} 2 a Q_{2} z_{1, k}^{2}\left\langle X_{1}^{x_{0}}(t), e_{k}\right\rangle_{L^{2}(D)} \delta_{k, n} \\
= & (I)+(I I)+(I I I), \\
& \left\langle\left(Q_{1} E_{4}-Q_{2} F_{4}\right) X_{2}^{x_{0}}(t), e_{n}\right\rangle_{L^{2}(D)}= \\
= & \sum_{k=1}^{\infty}\left(Q_{1} z_{2, k}^{2}-Q_{2} z_{1, k}^{2}\right)\left\langle X_{2}^{x_{0}}(t), e_{k}\right\rangle_{L^{2}(D)} \delta_{k, n} \\
& +\sum_{k=1}^{\infty} \sum_{l=k+1}^{\infty} \frac{8 a^{2}}{D_{k, l}}\left(Q_{1} z_{2, k} z_{2, l}\left(\alpha_{k}+\alpha_{l}\right)-2 Q_{2} z_{1, k} z_{1, l} \sqrt{\alpha_{k} \alpha_{l}}\right)\left\langle X_{2}^{x_{0}}(t), e_{k}\right\rangle_{L^{2}(D)} \delta_{n, l} \\
& +\sum_{k=1}^{\infty} \sum_{l=k+1}^{\infty} \frac{8 a^{2}}{D_{k, l}}\left(Q_{1} z_{2, k} z_{2, l}\left(\alpha_{k}+\alpha_{l}\right)-2 Q_{2} z_{1, k} z_{1, l} \sqrt{\alpha_{k} \alpha_{l}}\right)\left\langle X_{2}^{x_{0}}(t), e_{l}\right\rangle_{L^{2}(D)} \delta_{k, n} \\
= & (I V)+(V)+(V I) .
\end{aligned}
$$

By the central limit theorem for martingales, Law $\left(w_{1}(T)\right)$ converges weakly to a Gaussian distribution with a zero mean and variance given by the $\mathbb{P}-$ a.s. limit

$$
\lim _{T \rightarrow \infty} \frac{1}{T} \int_{0}^{T} \sum_{n=1}^{\infty} \lambda_{n}((I)+\ldots+(V I))^{2} d t .
$$

The limits of the cross terms are zero due to Lemma4.4 (see the proof of Theorem 4.6). We compute the limits of the "diagonal" terms in the $\mathbb{P}-$ a.s. sense. 


$$
\begin{aligned}
(A) & =\lim _{T \rightarrow \infty} \frac{1}{T} \int_{0}^{T} \sum_{n=1}^{\infty} \lambda_{n}(I)^{2} d t \\
& =\lim _{T \rightarrow \infty} \frac{1}{T} \int_{0}^{T} \sum_{n=1}^{\infty} \lambda_{n}\left(\sum_{l=n+1}^{\infty} \frac{4 a}{D_{n, l}}(\cdots)\left\langle X_{1}^{x_{0}}(t), e_{l}\right\rangle_{L^{2}(D)}\right)^{2} d t \\
& \stackrel{(*)}{=} \lim _{T \rightarrow \infty} \frac{1}{T} \int_{0}^{T} \sum_{n=1}^{\infty} \sum_{l=n+1}^{\infty} \lambda_{n} \frac{16 a^{2}}{D_{n, l}^{2}}(\cdots)^{2}\left\langle X_{1}^{x_{0}}(t), e_{l}\right\rangle_{L^{2}(D)}^{2} d t \\
& =\sum_{n=1}^{\infty} \sum_{l=n+1}^{\infty} \frac{1}{D_{n, l}^{2}} \frac{4 a \lambda_{n} \lambda_{l}}{b \alpha_{l}} \times \\
& \left(Q_{1} z_{2, n} z_{2, l} b \alpha_{l}\left(\alpha_{n}-\alpha_{l}\right)-Q_{2} z_{1, n} z_{1, l} \sqrt{\alpha_{n} \alpha_{l}}\left(8 a^{2}+b\left(\alpha_{n}-\alpha_{l}\right)\right)\right)^{2},
\end{aligned}
$$

since $\lim _{T \rightarrow \infty} \frac{1}{T} \int_{0}^{T}\left\langle X_{1}^{x_{0}}(t), e_{l}\right\rangle_{L^{2}(D)}^{2} d t=\frac{\lambda_{l}}{4 a b \alpha_{l}}$ and in the equality $(*)$ we have also used Lemma 4.4 for the cross summands. ( $\cdots)$ stands for the bracket from the definition of $(I)$.

Similarly, we have

$$
\begin{aligned}
& (B)=\lim _{T \rightarrow \infty} \frac{1}{T} \int_{0}^{T} \sum_{n=1}^{\infty} \lambda_{n}(I I)^{2} d t \\
& =\lim _{T \rightarrow \infty} \frac{1}{T} \int_{0}^{T} \sum_{n=1}^{\infty} \lambda_{n}\left(\sum_{k=1}^{n-1} \frac{4 a}{D_{k, n}}(\cdots)\left\langle X_{1}^{x_{0}}(t), e_{k}\right\rangle_{L^{2}(D)}\right)^{2} d t \\
& \stackrel{(*)}{=} \lim _{T \rightarrow \infty} \frac{1}{T} \int_{0}^{T} \sum_{n=1}^{\infty} \sum_{k=1}^{n-1} \lambda_{n} \frac{16 a^{2}}{D_{k, n}^{2}}(\cdots)^{2}\left\langle X_{1}^{x_{0}}(t), e_{k}\right\rangle_{L^{2}(D)}^{2} d t \\
& =\sum_{n=1}^{\infty} \sum_{k=1}^{n-1} \frac{1}{D_{k, n}^{2}} \frac{4 a \lambda_{n} \lambda_{k}}{b \alpha_{k}} \times \\
& \left(Q_{1} z_{2, k} z_{2, n} b \alpha_{k}\left(\alpha_{n}-\alpha_{k}\right)-Q_{2} z_{1, k} z_{1, n} \sqrt{\alpha_{k} \alpha_{n}}\left(8 a^{2}+b\left(\alpha_{n}-\alpha_{k}\right)\right)\right)^{2}, \\
& (C)=\lim _{T \rightarrow \infty} \frac{1}{T} \int_{0}^{T} \sum_{n=1}^{\infty} \lambda_{n}(I I I)^{2} d t \\
& =\lim _{T \rightarrow \infty} \frac{1}{T} \int_{0}^{T} \sum_{n=1}^{\infty} 4 a^{2} Q_{2}^{2} \lambda_{n} z_{1, n}^{4}\left\langle X_{1}^{x_{0}}(t), e_{n}\right\rangle_{L^{2}(D)}^{2} d t \\
& =\frac{a Q_{2}^{2}}{b} \sum_{n=1}^{\infty} \frac{\lambda_{n}^{2}}{\alpha_{n}} z_{1, n}^{4} \\
& (D)=\lim _{T \rightarrow \infty} \frac{1}{T} \int_{0}^{T} \sum_{n=1}^{\infty} \lambda_{n}(I V)^{2} d t \\
& =\lim _{T \rightarrow \infty} \frac{1}{T} \int_{0}^{T} \sum_{n=1}^{\infty} \lambda_{n}\left(Q_{1} z_{2, n}^{2}-Q_{2} z_{1, n}^{2}\right)^{2}\left\langle X_{2}^{x_{0}}(t), e_{n}\right\rangle_{L^{2}(D)}^{2} d t \\
& =\frac{1}{4 a} \sum_{n=1}^{\infty} \lambda_{n}^{2}\left(Q_{1} z_{2, n}^{2}-Q_{2} z_{1, n}^{2}\right)^{2} \text {, }
\end{aligned}
$$


since $\lim _{T \rightarrow \infty} \frac{1}{T} \int_{0}^{T}\left\langle X_{2}^{x_{0}}(t), e_{n}\right\rangle_{L^{2}(D)}^{2} d t=\frac{\lambda_{n}}{4 a}$.

Next, we have

$$
\begin{aligned}
(E) & =\lim _{T \rightarrow \infty} \frac{1}{T} \int_{0}^{T} \sum_{n=1}^{\infty} \lambda_{n}(V)^{2} d t \\
& =\lim _{T \rightarrow \infty} \frac{1}{T} \int_{0}^{T} \sum_{n=1}^{\infty} \lambda_{n}\left(\sum_{k=1}^{n-1} \frac{8 a^{2}}{D_{k, n}}(\cdots)\left\langle X_{2}^{x_{0}}(t), e_{k}\right\rangle_{L^{2}(D)}\right)^{2} d t \\
& \stackrel{(*)}{=} \lim _{T \rightarrow \infty} \frac{1}{T} \int_{0}^{T} \sum_{n=1}^{\infty} \sum_{k=1}^{n-1} \lambda_{n} \frac{64 a^{4}}{D_{k, n}^{2}}(\cdots)^{2}\left\langle X_{2}^{x_{0}}(t), e_{k}\right\rangle_{L^{2}(D)}^{2} d t \\
& =\sum_{n=1}^{\infty} \sum_{k=1}^{n-1} \frac{1}{D_{k, n}^{2}} 16 a^{3} \lambda_{n} \lambda_{k}\left(Q_{1} z_{2, k} z_{2, n}\left(\alpha_{k}+\alpha_{n}\right)-2 Q_{2} z_{1, k} z_{1, n} \sqrt{\alpha_{k} \alpha_{n}}\right)^{2}, \\
& =\lim _{T \rightarrow \infty} \frac{1}{T} \int_{0}^{T} \sum_{n=1}^{\infty} \lambda_{n}(V I)^{2} d t \\
& =\lim _{T \rightarrow \infty} \frac{1}{T} \int_{0}^{T} \sum_{n=1}^{\infty} \lambda_{n}\left(\sum_{l=n+1}^{\infty} \frac{8 a^{2}}{D_{n, l}}(\cdots)\left\langle X_{2}^{x_{0}}(t), e_{l}\right\rangle_{L^{2}(D)}\right)^{2} d t \\
& \stackrel{(*)}{=} \lim _{T \rightarrow \infty} \frac{1}{T} \int_{0}^{T} \sum_{n=1}^{\infty} \sum_{l=n+1}^{\infty} \lambda_{n} \frac{64 a^{4}}{D_{n, l}^{2}}(\cdots)^{2}\left\langle X_{2}^{x_{0}}(t), e_{l}\right\rangle_{L^{2}(D)}^{2} d t \\
& =\sum_{n=1}^{\infty} \sum_{l=n+1}^{\infty} \frac{1}{D_{n, l}^{2} 16 a^{3} \lambda_{n} \lambda_{l}\left(Q_{1} z_{2, n} z_{2, l}\left(\alpha_{n}+\alpha_{l}\right)-2 Q_{2} z_{1, n} z_{1, l} \sqrt{\alpha_{n} \alpha_{l}}\right)^{2} .}
\end{aligned}
$$

The limiting variance of $w_{1}(T)$ is the sum of the six above terms $(A)+\ldots+(F)$, which can be simplified (analogously as in the proof of Theorem 4.6) to

$$
\begin{aligned}
& \frac{16 a^{3} b Q_{2}^{2}}{b} \sum_{k=1}^{\infty} \sum_{n=1}^{\infty} \frac{\lambda_{k} \lambda_{n} z_{1, k}^{2} z_{1, n}^{2}}{D_{k, n}} \\
& +2 a \sum_{k=1}^{\infty} \sum_{n=1}^{\infty} \frac{\lambda_{k} \lambda_{n}}{D_{k, n}}\left(\left(Q_{1} z_{2, k} z_{2, n} \sqrt{\alpha_{k}}-Q_{2} z_{1, k} z_{1, n} \sqrt{\alpha_{n}}\right)^{2}\right. \\
& \left.+\left(Q_{1} z_{2, k} z_{2, n} \sqrt{\alpha_{n}}-Q_{2} z_{1, k} z_{1, n} \sqrt{\alpha_{k}}\right)^{2}\right) .
\end{aligned}
$$

Since the multiplicative factor $\frac{1}{2 a Q_{2} \frac{1}{T} \int_{0}^{T}\left\langle X_{1}^{x_{0}}(t), z_{1}\right\rangle_{\operatorname{Dom}\left((-A)^{\frac{1}{2}}\right)}^{2}}$ of $w_{1}(T)$ on the right-hand side of (4.17) converges to $\frac{2 b}{Q_{1} Q_{2}}$ as $T \rightarrow \infty$, we arrive at (4.16).

4.3. Asymptotic normality of the estimator $\bar{b}_{T, z_{1}, a}$. Based on the estimation strategy 2., another estimator of the parameter $b$ may be introduced. Using "observation window" $\left(z_{1}, 0\right)^{\top}, z_{1} \neq 0$, with the parameter $a$ known, we may define

$$
\bar{b}_{T, z_{1}, a}=\frac{\left\langle Q z_{1}, z_{1}\right\rangle_{\operatorname{Dom}\left((-A)^{\frac{1}{2}}\right)}}{4 a \frac{1}{T} \int_{0}^{T}\left\langle X_{1}^{x_{0}}(t), z_{1}\right\rangle_{\operatorname{Dom}\left((-A)^{\frac{1}{2}}\right)}^{2} d t} .
$$

This estimator $\bar{b}_{T, z_{1}, a}$ is also strongly consistent as $T \rightarrow \infty$ by Theorem 3.2 and we show its asymptotic normality. 
Theorem 4.11. Let $0 \neq z_{1} \in \operatorname{Dom}\left((-A)^{\frac{1}{2}}\right), a>0$ be arbitrary. The estimator $\bar{b}_{T, z_{1}, a}$ is asymptotically normal, i.e., Law $\left(\sqrt{T}\left(\bar{b}_{T, z_{1}, a}-b\right)\right)$ converges weakly to a centered Gaussian distribution with variance given by

$$
\begin{aligned}
& \frac{64 a^{3} b}{Q_{1}^{2}} \sum_{k=1}^{\infty} \sum_{n=1}^{\infty} \frac{\lambda_{k} \lambda_{n} z_{1, k}^{2} z_{1, n}^{2}}{b\left(\alpha_{k}-\alpha_{n}\right)^{2}+8 a^{2}\left(\alpha_{k}+\alpha_{n}\right)} \\
& +\frac{8 a b^{2}}{Q_{1}^{2}} \sum_{k=1}^{\infty} \sum_{n=1}^{\infty} \frac{\lambda_{k} \lambda_{n}\left(\alpha_{k}+\alpha_{n}\right) z_{1, k}^{2} z_{1, n}^{2}}{b\left(\alpha_{k}-\alpha_{n}\right)^{2}+8 a^{2}\left(\alpha_{k}+\alpha_{n}\right)} .
\end{aligned}
$$

Proof. Set $0 \neq z_{1} \in \operatorname{Dom}\left((-A)^{\frac{1}{2}}\right)$. Using formula (4.18) for the estimator $\bar{b}_{T, z_{1}, a}$ and Lemma 4.9, we obtain

$$
\begin{aligned}
& \sqrt{T}\left(\bar{b}_{T, z_{1}, a}-b\right)= \\
& =\frac{\sqrt{T}}{4 a \frac{1}{T} \int_{0}^{T}\left\langle X_{1}^{x_{0}}(t), z_{1}\right\rangle_{\operatorname{Dom}\left((-A)^{\frac{1}{2}}\right)}^{2} d t}\left(Q_{1}-\frac{4 a b}{T} \int_{0}^{T}\left\langle X_{1}^{x_{0}}(t), z_{1}\right\rangle_{\operatorname{Dom}\left((-A)^{\frac{1}{2}}\right)}^{2} d t\right) \\
& =\frac{1}{4 a \frac{1}{T} \int_{0}^{T}\left\langle X_{1}^{x_{0}}(t), z_{1}\right\rangle_{\operatorname{Dom}\left((-A)^{\frac{1}{2}}\right)}^{2} d t} \frac{1}{\sqrt{T}}\left(\left\langle F X^{x_{0}}(T), X^{x_{0}}(T)\right\rangle_{V}-\left\langle F x_{0}, x_{0}\right\rangle_{V}\right) \\
& -\frac{1}{2 a \frac{1}{T} \int_{0}^{T}\left\langle X_{1}^{x_{0}}(t), z_{1}\right\rangle_{\operatorname{Dom}\left((-A)^{\frac{1}{2}}\right)}^{2} d t} \frac{1}{\sqrt{T}} \int_{0}^{T}\left\langle F X^{x_{0}}(t), \Phi d B(t)\right\rangle_{V} .
\end{aligned}
$$

The first term converges to zero in probability as $T \rightarrow \infty$ (see the proof of Theorem 4.10) and define

$$
\begin{aligned}
w_{2}(T) & =\frac{1}{\sqrt{T}} \int_{0}^{T}\left\langle F X^{x_{0}}(t), \Phi d B(t)\right\rangle_{V} \\
& =\frac{1}{\sqrt{T}} \int_{0}^{T} \sum_{n=1}^{\infty} \sqrt{\lambda_{n}}\left\langle F X^{x_{0}}(t),\left(\begin{array}{c}
0 \\
e_{n}
\end{array}\right)\right\rangle_{V} d \beta_{n}(t) .
\end{aligned}
$$

Since the stochastic integral $w_{2}(T)$ is a special case of the stochastic integral $w_{1}(T)$ from the proof of Theorem 4.10 (use $E=0, Q_{2}=1$ and omit the minus sign), we will handle it much easier than above. The required scalar products equal to

$$
\begin{aligned}
& \left\langle F_{3} X_{1}^{x_{0}}(t), e_{n}\right\rangle_{L^{2}(D)}= \\
& =\sum_{k=1}^{\infty} \sum_{l=k+1}^{\infty} \frac{z_{1, k} z_{1, l}}{D_{k, l}} 4 a \sqrt{\alpha_{k} \alpha_{l}}\left(8 a^{2}+b\left(\alpha_{k}-\alpha_{l}\right)\right)\left\langle X_{1}^{x_{0}}(t), e_{l}\right\rangle_{L^{2}(D)} \delta_{k, n} \\
& \quad+\sum_{k=1}^{\infty} \sum_{l=k+1}^{\infty} \frac{z_{1, k} z_{1, l}}{D_{k, l}} 4 a \sqrt{\alpha_{k} \alpha_{l}}\left(8 a^{2}+b\left(\alpha_{l}-\alpha_{k}\right)\right)\left\langle X_{1}^{x_{0}}(t), e_{k}\right\rangle_{L^{2}(D)} \delta_{n, l} \\
& \quad+\sum_{k=1}^{\infty} 2 a z_{1, k}^{2}\left\langle X_{1}^{x_{0}}(t), e_{k}\right\rangle_{L^{2}(D)} \delta_{k, n} \\
& =(I)+(I I)+(I I I),
\end{aligned}
$$




$$
\begin{aligned}
& \left\langle F_{4} X_{2}^{x_{0}}(t), e_{n}\right\rangle_{L^{2}(D)}= \\
& =\sum_{k=1}^{\infty} z_{1, k}^{2}\left\langle X_{2}^{x_{0}}(t), e_{k}\right\rangle_{L^{2}(D)} \delta_{k, n} \\
& \quad+\sum_{k=1}^{\infty} \sum_{l=k+1}^{\infty} \frac{z_{1, k} z_{1, l}}{D_{k, l}} 16 a^{2} \sqrt{\alpha_{k} \alpha_{l}}\left\langle X_{2}^{x_{0}}(t), e_{k}\right\rangle_{L^{2}(D)} \delta_{n, l} \\
& \quad+\sum_{k=1}^{\infty} \sum_{l=k+1}^{\infty} \frac{z_{1, k} z_{1, l}}{D_{k, l}} 16 a^{2} \sqrt{\alpha_{k} \alpha_{l}}\left\langle X_{2}^{x_{0}}(t), e_{l}\right\rangle_{L^{2}(D)} \delta_{k, n} \\
& =(I V)+(V)+(V I) .
\end{aligned}
$$

The appropriate limits of the "diagonal" terms equal to

$$
\begin{aligned}
& (A)=\lim _{T \rightarrow \infty} \frac{1}{T} \int_{0}^{T} \sum_{n=1}^{\infty} \lambda_{n}(I)^{2} d t=\sum_{n=1}^{\infty} \sum_{l=n+1}^{\infty} \frac{z_{1, n}^{2} z_{1, l}^{2}}{D_{n, l}^{2}} \frac{4 a \lambda_{n} \lambda_{l} \alpha_{n}}{b}\left(8 a^{2}+b\left(\alpha_{n}-\alpha_{l}\right)\right)^{2} \\
& (B)=\lim _{T \rightarrow \infty} \frac{1}{T} \int_{0}^{T} \sum_{n=1}^{\infty} \lambda_{n}(I I)^{2} d t=\sum_{n=1}^{\infty} \sum_{k=1}^{n-1} \frac{z_{1, k}^{2} z_{1, n}^{2}}{D_{k, n}^{2}} \frac{4 a \lambda_{n} \lambda_{k} \alpha_{n}}{b}\left(8 a^{2}+b\left(\alpha_{n}-\alpha_{k}\right)\right)^{2}, \\
& (C)=\lim _{T \rightarrow \infty} \frac{1}{T} \int_{0}^{T} \sum_{n=1}^{\infty} \lambda_{n}(I I I)^{2} d t=\frac{a}{b} \sum_{n=1}^{\infty} \frac{\lambda_{n}^{2}}{\alpha_{n}} z_{1, n}^{4}, \\
& (D)=\lim _{T \rightarrow \infty} \frac{1}{T} \int_{0}^{T} \sum_{n=1}^{\infty} \lambda_{n}(I V)^{2} d t=\frac{1}{4 a} \sum_{n=1}^{\infty} \lambda_{n}^{2} z_{1, n}^{4}, \\
& (E)=\lim _{T \rightarrow \infty} \frac{1}{T} \int_{0}^{T} \sum_{n=1}^{\infty} \lambda_{n}(V)^{2} d t=\sum_{n=1}^{\infty} \sum_{k=1}^{n-1} \frac{z_{1, k}^{2} z_{1, n}^{2}}{D_{k, n}^{2}} 64 a^{3} \lambda_{n} \lambda_{k} \alpha_{n} \alpha_{k}, \\
& (F)=\lim _{T \rightarrow \infty} \frac{1}{T} \int_{0}^{T} \sum_{n=1}^{\infty} \lambda_{n}(V I)^{2} d t=\sum_{n=1}^{\infty} \sum_{l=n+1}^{\infty} \frac{z_{1, n}^{2} z_{1, l}^{2}}{D_{n, l}^{2}} 64 a^{3} \lambda_{n} \lambda_{l} \alpha_{n} \alpha_{l} .
\end{aligned}
$$

Analogously as above, Law $\left(w_{2}(T)\right)$ converges weakly to a Gaussian distribution with a zero mean and variance given by $(A)+\ldots+(F)$, which can be simplified to

$$
\frac{16 a^{3}}{b} \sum_{k=1}^{\infty} \sum_{n=1}^{\infty} \frac{\lambda_{k} \lambda_{n} z_{1, k}^{2} z_{1, n}^{2}}{D_{k, n}}+2 a \sum_{k=1}^{\infty} \sum_{n=1}^{\infty} \frac{\lambda_{k} \lambda_{n}\left(\alpha_{k}+\alpha_{n}\right) z_{1, k}^{2} z_{1, n}^{2}}{D_{k, n}} .
$$

Since the multiplicative factor of $w_{2}(T)$ on the right-hand side of (4.20) converges to $-\frac{2 b}{Q_{1}}$, we arrive at (4.19).

Remark 4.12. If we consider some special cases of "observation windows", the formulae for the limiting variances from Theorems 4.6, 4.10 and 4.11 may be considerably simplified.

- The estimator $\bar{a}_{T, k}$ (defined by (3.6) ) has limiting variance $a$ for any $k \in \mathbb{N}$, i.e.,

$$
\text { Law }\left(\sqrt{T}\left(\bar{a}_{T, k}-a\right)\right) \stackrel{w^{*}}{\longrightarrow} N(0, a), \quad T \rightarrow \infty .
$$

Since the result does not depend on $k$ it does not matter which coordinate of the second component we observe. All the estimators $\bar{a}_{T, k}$ behave similarly. 
- The estimator $\bar{b}_{T, j, k}$ (defined by (3.77) satisfies

$$
\operatorname{Law}\left(\sqrt{T}\left(\bar{b}_{T, j, k}-b\right)\right) \stackrel{w^{*}}{\longrightarrow} N\left(0, \frac{4 a b}{\alpha_{j}}+\frac{2 b^{2}}{a}\right), \quad T \rightarrow \infty,
$$

for any $j, k \in \mathbb{N}, j \neq k$.

- However, if $j=k$ then the estimator $\bar{b}_{T, j, j}$ satisfies

$$
\text { Law }\left(\sqrt{T}\left(\bar{b}_{T, j, j}-b\right)\right) \stackrel{w^{*}}{\longrightarrow} N\left(0, \frac{4 a b}{\alpha_{j}}\right), \quad T \rightarrow \infty .
$$

- The estimator $\bar{b}_{T, f_{j}, a}$ (defined by (4.18)) satisfies

$$
\operatorname{Law}\left(\sqrt{T}\left(\bar{b}_{T, f_{j}, a}-b\right)\right) \stackrel{w^{*}}{\longrightarrow} N\left(0, \frac{4 a b}{\alpha_{j}}+\frac{b^{2}}{a}\right), \quad T \rightarrow \infty,
$$

for any $j \in \mathbb{N}$.

By comparing the last three points, we obtain two following observations, which are illustrated by simulations in Section 6 .

1. Since the limiting variance of the estimator $\bar{b}_{T, j, k}$ is greater than the limiting variance of the estimator $\bar{b}_{T, f_{j}, a}$, it seems that it is better to know the true value of the parameter $a$ exactly, instead of estimating it. However, if $j=k$ then the limiting variance of the estimator $\bar{b}_{T, j, j}$ is even smaller. So estimating the parameter $a$ by the "window" $\left(0, e_{j}\right)^{\top}$ and then using the "window" $\left(f_{j}, 0\right)^{\top}$ to estimate the parameter $b$ should be even better than knowing $a$ exactly.

2. Since $\alpha_{j} \rightarrow \infty$ as $j \rightarrow \infty$, the limiting variance $\frac{4 a b}{\alpha_{j}}$ gets smaller with bigger $j$. Hence it is better to use the "observation coordinate" with bigger $j$ rather than using the smaller one.

\section{EXAMPLE}

Example 5.1. Consider the stochastic wave equation with Dirichlet boundary conditions

$$
\begin{aligned}
\frac{\partial^{2} u}{\partial t^{2}}(t, \xi) & =b \Delta u(t, \xi)-2 a \frac{\partial u}{\partial t}(t, \xi)+\eta(t, \xi), \quad(t, \xi) \in \mathbb{R}_{+} \times D, \\
u(0, \xi) & =u_{1}(\xi), \quad \xi \in D \\
\frac{\partial u}{\partial t}(0, \xi) & =u_{2}(\xi), \quad \xi \in D \\
u(t, \xi) & =0, \quad(t, \xi) \in \mathbb{R}_{+} \times \partial D
\end{aligned}
$$

where $D \subset \mathbb{R}^{d}$ is a bounded domain with a smooth boundary, $\eta$ is a noise process that is the formal time derivative of a space dependent Brownian motion and $a>0$, $b>0$ are unknown parameters.

We rewrite the hyperbolic system (5.1) as an infinite dimensional stochastic differential equation (2.2)

$$
\begin{gathered}
d X(t)=\mathcal{A} X(t) d t+\Phi d B(t), \\
X(0)=x_{0}=\left(\begin{array}{l}
u_{1} \\
u_{2}
\end{array}\right)
\end{gathered}
$$


for $t \geqslant 0$, setting $A=\left.\Delta\right|_{\operatorname{Dom}(A)}, \operatorname{Dom}(A)=H^{2}(D) \cap H_{0}^{1}(D), \operatorname{Dom}(\mathcal{A})=\operatorname{Dom}(A) \times$ $\operatorname{Dom}\left((-A)^{\frac{1}{2}}\right)$ and

$$
\mathcal{A}=\left(\begin{array}{cc}
0 & I \\
b A & -2 a I
\end{array}\right)
$$

The operator $\mathcal{A}$ generates strongly continuous semigroup in the space $V=$ $\operatorname{Dom}\left((-A)^{\frac{1}{2}}\right) \times L^{2}(D)$. The driving process may take a form $B(t)=(0, \tilde{B}(t))^{\top}$, where $(\tilde{B}(t), t \geqslant 0)$ is a standard cylindrical Brownian motion on $L^{2}(D)$. The noise $\eta$ is modelled as the formal derivative $\Phi_{1} \frac{d \tilde{B}(t)}{d t}, \Phi_{1} \in \mathcal{L}_{2}\left(L^{2}(D)\right)$ and $\Phi \in \mathcal{L}_{2}(V)$ is given by

$$
\Phi=\left(\begin{array}{cc}
0 & 0 \\
0 & \Phi_{1}
\end{array}\right) .
$$

With this setup, all assumptions of Section 2 are fulfilled, so Theorem 3.2 may be used for estimation of parameters. Theorems 4.6, 4.10 and 4.11, which show asymptotic normality of these estimators, may be applied as well.

\section{IMPLEMENTATION AND STATISTICAL EVIDENCE}

We have generated a trajectory of the solution to the stochastic differential equation (5.1) from Example 5.1 in the program R by Euler's method (see [3]). The setup of Example 5.1 is specified as follows:

- $D=(0,1)$ - We consider the wave equation for the oscillating rod modelled as a function from the space $L^{2}((0,1))$.

- The choice of the orthonormal basis of the space $L^{2}((0,1))$ is

$$
\left\{e_{n}(\xi)=\sqrt{2} \sin (n \pi \xi), n=1, \ldots, N\right\},
$$

whose elements satisfy the boundary condition $u(t, 0)=0=u(t, 1)$ for any $t>0$.

- $N=10-$ We have restricted the expansion of the previous basis only to $N=$ 10 functions. The accuracy of our results may suffer due to this limitation, nevertheless we will show that our results are sufficiently satisfactory.

- $T=1000$ - The length of the time interval.

- $\Delta t=0.0001-$ The mesh of the partition of the time interval $[0, T]$.

- The intial functions $u_{1}$ and $u_{2}$ have the following form

$$
u_{1}(\xi)=\sqrt{2} \sum_{n=1}^{N} \sin (n \pi \xi)=u_{2}(\xi)
$$

This means that $\left\langle u_{1}, e_{n}\right\rangle_{L^{2}(D)}=1=\left\langle u_{2}, e_{n}\right\rangle_{L^{2}(D)}$ for any $n=1, \ldots, N$, so the initial conditions are the same in all $N$ dimensions.

- $a=1, b=0.2$ - The values of the parameters that are to be estimated.

- $-\alpha_{n}=-n^{2} \pi^{2}$ - The eigenvalues of the operator $A$. With this setup the operator $A$ is the Laplacian operator $A=\left.\Delta\right|_{\operatorname{Dom}(A)}$ with $\operatorname{Dom}(A)=$ $H^{2}((0,1)) \cap H_{0}^{1}((0,1))$.

- $\lambda_{n}=\frac{1000}{n^{2}}$ - The eigenvalues of the operator $Q$. (The eigenvalues of the operator $\Phi_{1}$ equal to $\sqrt{\lambda_{n}}$ for any $n=1, \ldots, N$.) The eigenvalues are chosen in the way that the sum $\sum_{n=1}^{\infty} \lambda_{n}$ is convergent. The multiplication factor is chosen in order to increase the values of the $\lambda_{n}$. Otherwise the noise would be in "higher" dimensions so small that it would be practically vanishing. 
We will focus on the estimation of the parameter $a$ using the first and tenth coordinate of the second component, i.e., $\bar{a}_{T, k=1}$ and $\bar{a}_{T, k=10}$ (see (3.6)), and the estimation of the parameter $b$ using the estimators $\bar{a}_{T, k=1}$ and $\bar{a}_{T, k=10}$ of $a$ together with the first and tenth coordinate of the second component, respectively, i.e., $\bar{b}_{T, j=1, k=1}$ and $\bar{b}_{T, j=10, k=10}$ (see (3.7)). We will also study the estimators $\bar{b}_{T, j=1, a=1}$ and $\bar{b}_{T, j=10, a=1}$, which depend only on the "observation window" $\left(f_{1}, 0\right)^{\top}$ (or $\left.\left(f_{10}, 0\right)^{\top}\right)$ with the parameter $a$ supposed to be known (see (4.18) ).

Using the generated trajectory, we obtained the following results:

$$
\begin{array}{llll}
\bar{a}_{T, k=1} & =0.9994, & \bar{a}_{T, k=10} & =0.9978, \\
\bar{b}_{T, j=1, k=1} & =0.1902, & \bar{b}_{T, j=10, k=10} & =0.2001, \\
\bar{b}_{T, j=1, a=1} & =0.1901, & \bar{b}_{T, j=10, a=1} & =0.1997 .
\end{array}
$$

Time evolution of these estimators is depicted in Figures 1,2 and 3.

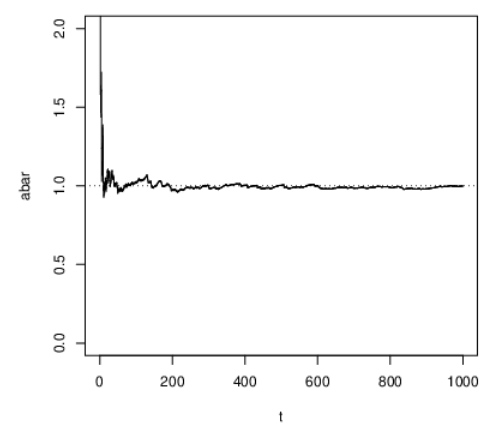

(a) The estimator $\bar{a}_{t, k=1}$

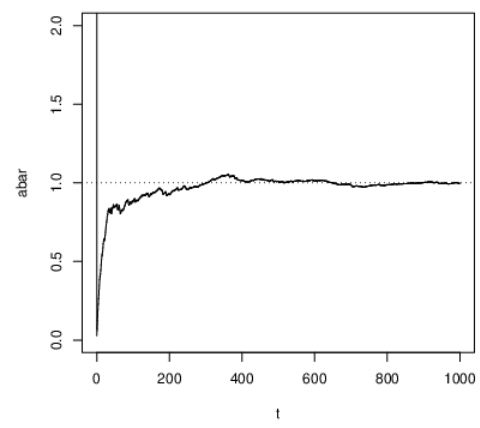

(b) The estimator $\bar{a}_{t, k=10}$

Figure 1 . The time evolution of the estimators $\bar{a}_{t, k}$ for $k=1$ and $k=10$

Although the results seems satisfactory (especially for the estimator $\bar{b}_{T, j=10, k=10}$ ), we have made 100 more simulations in a similar manner. The values of the estimators $\bar{a}_{T, k=1}$ and $\bar{a}_{T, k=10}$ are depicted in Figure 4 and the values of the estimators $\bar{b}_{T, j=1, k=1}$ and $\bar{b}_{T, j=10, k=10}$ are depicted in Figure 5. Moreover, the values of the estimators $\bar{b}_{T, j=1, a=1}$ and $\bar{b}_{T, j=10, a=1}$ are shown in Figure 6. The overall statistics can be found in Tables 1 and 2 .

\begin{tabular}{|l||c|c|}
\hline & $\bar{a}_{T, k=1}$ & $\bar{a}_{T, k=10}$ \\
\hline \hline Mean & 0.9995 & 0.9673 \\
\hline Var & 1.1039 & 1.0036 \\
\hline Var - Theoretical & 1.0000 & 1.0000 \\
\hline Relative error - Maximal & $10 \%$ & $10 \%$ \\
\hline Relative error - Typical & $\leqslant 4 \%$ & $\leqslant 6 \%$ \\
\hline$p$-value & 0.9161 & 0.6340 \\
\hline
\end{tabular}

TABLE 1. The results of the simulations - Part I 


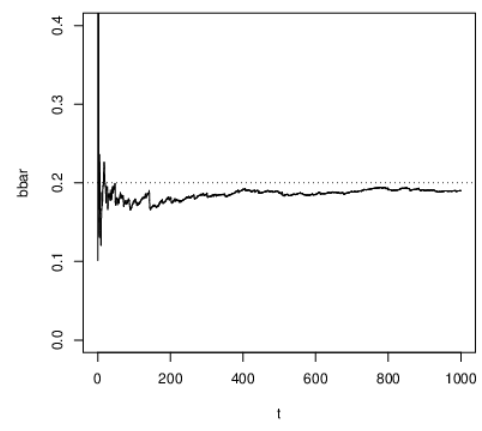

(a) The estimator $\bar{b}_{t, j=1, k=1}$

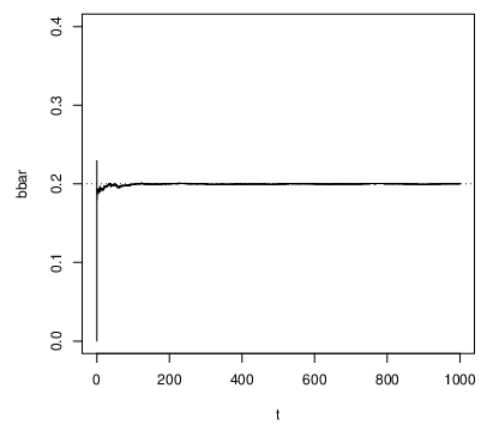

(b) The estimator $\bar{b}_{t, j=10, k=10}$

Figure 2. The time evolution of the estimators $\bar{b}_{t, j, k}$ for $j=k=1$ and $j=k=10$

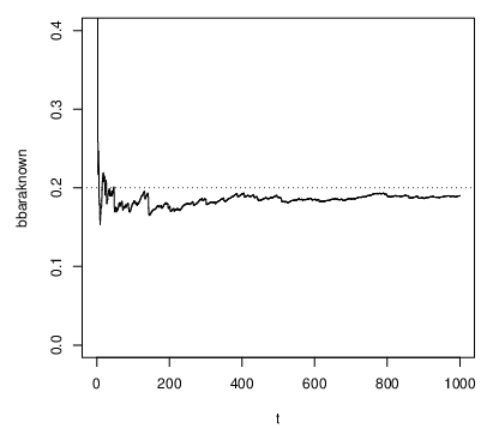

(a) The estimator $\bar{b}_{t, j=1, a=1}$

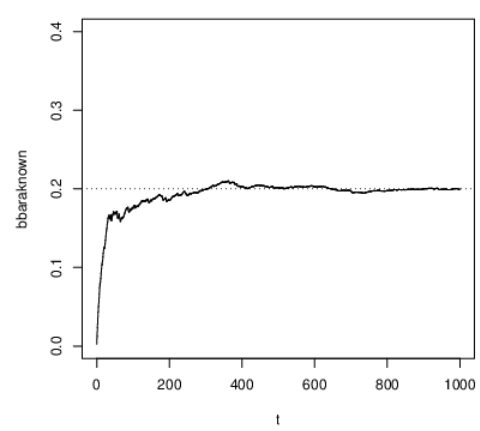

(b) The estimator $\bar{b}_{t, j=10, a=1}$

FiguRE 3 . The time evolution of the estimators $\bar{b}_{t, j, a}$ for $j=1$, $j=10$ and $a=1$

\begin{tabular}{|l||c|c|c|c|}
\hline & $b_{T, j=1, k=1}$ & $b_{T, j=10, k=10}$ & $b_{T, j=1, a=1}$ & $b_{T, j=10, a=1}$ \\
\hline \hline Mean & 0.1989 & 0.2000 & 0.1988 & 0.1935 \\
\hline Var & 0.0616 & 0.0008 & 0.1033 & 0.0402 \\
\hline Var - Theoretical & 0.0811 & 0.0008 & 0.1211 & 0.0408 \\
\hline Relative error - Maximal & $9 \%$ & $1 \%$ & $12 \%$ & $10 \%$ \\
\hline Relative error - Typical & $\leqslant 5 \%$ & $\leqslant 0.6 \%$ & $\leqslant 6 \%$ & $\leqslant 6 \%$ \\
\hline$p$-value & 0.7904 & 0.2986 & 0.2825 & 0.6668 \\
\hline
\end{tabular}

TABle 2. The results of the simulations - Part II

The row "Var" stands for the variance of $\sqrt{T}\left(\bar{a}_{T, k}-a\right)$ (and its analogues in the following columns). The actual variances of the estimators are 1000 times smaller. 


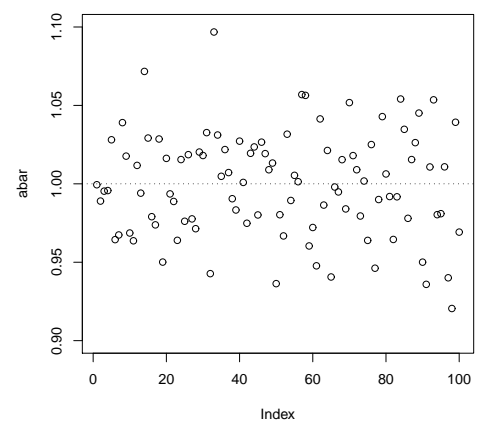

(a) The values of $\bar{a}_{T, k=1}-$ Overall

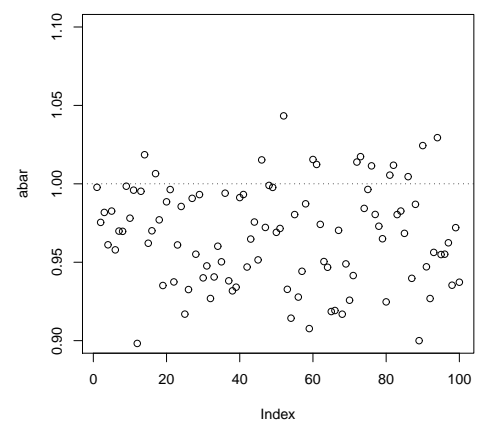

(b) The values of $\bar{a}_{T, k=10}$ - Overall

Figure 4 . The estimators $\bar{a}_{t, k}$ for $k=1$ and $k=10$ based on larger sample

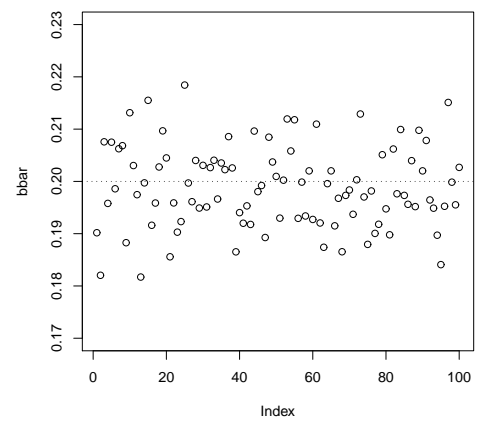

(a) The values of $\bar{b}_{T, j=1, k=1}$ - Overall

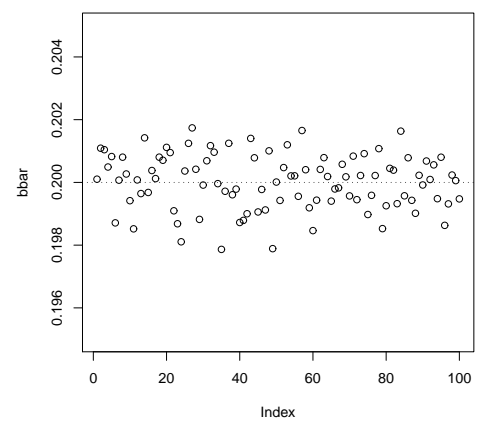

(b) The values of $\bar{b}_{T, j=10, k=10}$ - Overall

Figure 5. The estimators $\bar{b}_{t, j, k}$ for $j=k=1$ and $j=k=10$ based on larger sample

The theoretical values of the limiting variances (see formulae in Remark 4.12) can be found in the row "Var - Theoretical".

Since the absolute errors of the estimators can be viewed in Figures 4 , 5 and 6 we mention only relative errors: maximal (which is the relative error of the worst estimator) and typical (that is the level below which $75 \%$ of the errors belong).

The $p$-values of the Wilk-Shapiro test of normality can be found in the last row. Since they are greater than 0.05 , we do not reject the hypothesis of normality on $5 \%$-significance level. The Q-Q plots of the centered and rescaled estimators are shown in Figures 7,8 and 9 .

From the previous simulations the main three observations follow: 


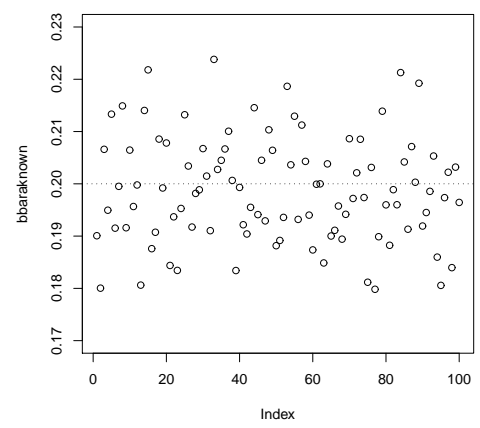

(a) The values of $\bar{b}_{T, j=1, a=1}$ - Overall

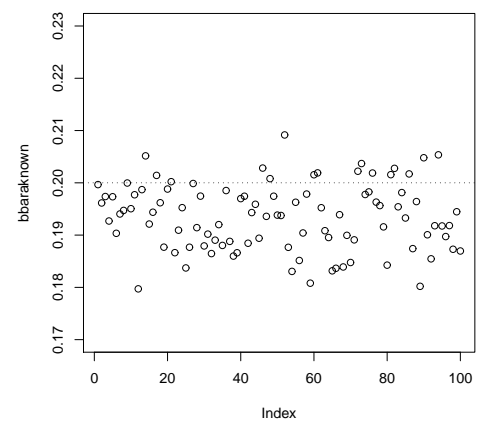

(b) The values of $\bar{b}_{T, j=10, a=1}$ - Overall

Figure 6 . The estimators $\bar{b}_{t, j, a}$ for $j=1, j=10$ and $a=1$ based on larger sample

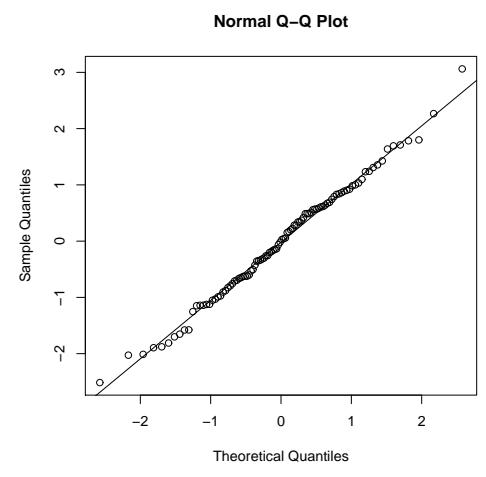

(a) Q-Q plot of $\sqrt{T}\left(\bar{a}_{T, k=1}-a\right)$

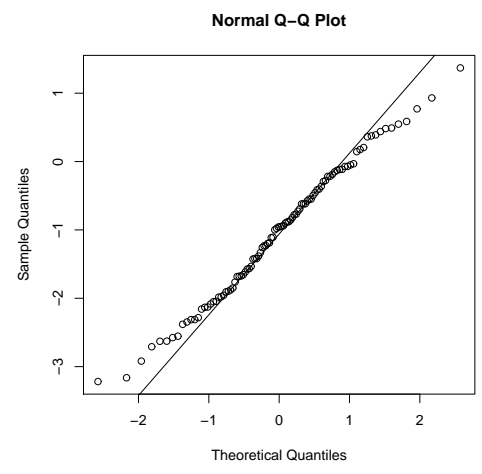

(b) Q-Q plot of $\sqrt{T}\left(\bar{a}_{T, k=10}-a\right)$

Figure 7. Asymptotic normality of $\bar{a}_{T, k}$ for $k=1$ and $k=10$

- The estimators $\bar{a}_{T, k=1}$ and $\bar{a}_{T, k=10}$ behave similarly (the estimator $\bar{a}_{T, k=10}$ would require some bigger time $T$, though), however there is a big difference between the estimators of the parameter $b$. Not only that the estimator $\bar{b}_{T, j=10, k=10}$ behaves better than the estimator $\bar{b}_{T, j=1, k=1}$ (it has better mean and lesser variance and relative errors), but also by comparing the estimator $\bar{b}_{T, j=1, k=1}$ with $\bar{b}_{T, j=1, a=1}$ (and $\bar{b}_{T, j=10, k=10}$ with $\bar{b}_{T, j=10, a=1}$ ), it seems that it is better to work with the paramater $a$ unknown. (See Remark 4.12.)

- From the comparing of the rows "Var" and "Var-Theoretical" it seems that the computed limiting variances from Remark 4.12 are accurate.

- From the Figures 78 and 9 and from the results of Wilk-Shapiro tests it seems that the estimators are asymptotically normal as prescribed by Theorems 4.6, 4.10 and 4.11. 


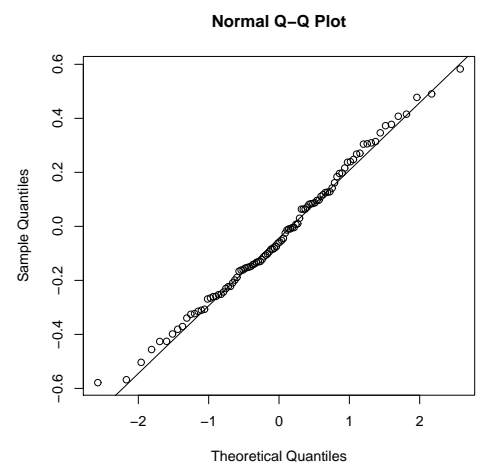

(a) Q-Q plot of $\sqrt{T}\left(\bar{b}_{T, j=1, k=1}-b\right)$

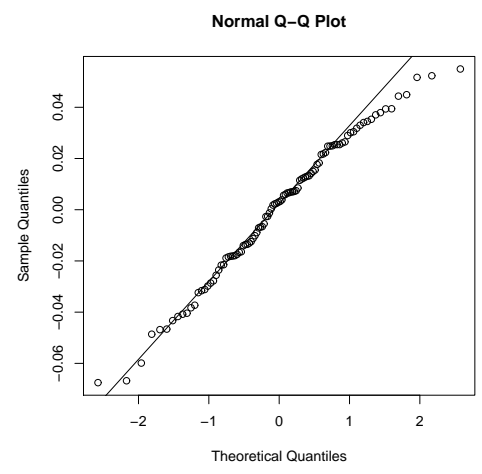

(b) Q-Q plot of $\sqrt{T}\left(\bar{b}_{T, j=10, k=10}-b\right)$

Figure 8. Asymptotic normality of $\bar{b}_{T, j, k}$ for $j=k=1$ and $j=k=10$

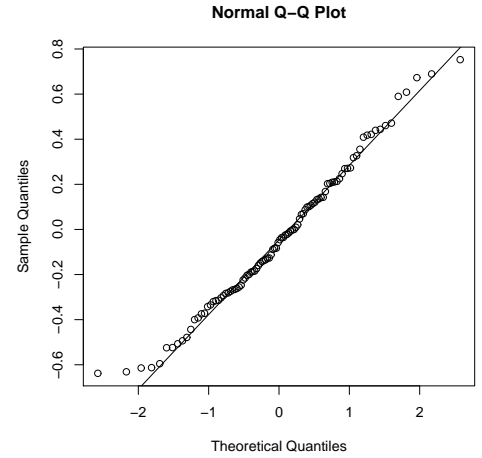

(a) Q-Q plot of $\sqrt{T}\left(\bar{b}_{T, j=1, a=1}-b\right)$

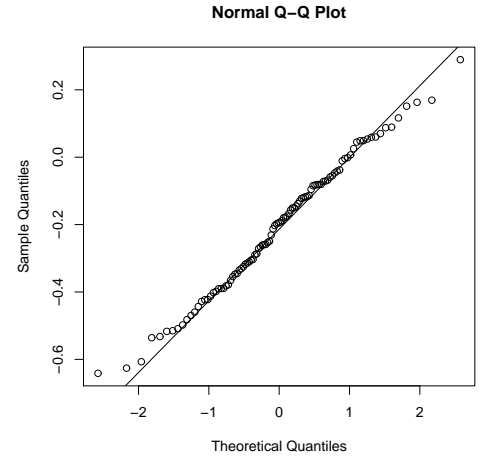

(b) Q-Q plot of $\sqrt{T}\left(\bar{b}_{T, j=10, a=1}-b\right)$

Figure 9. Asymptotic normality of $\bar{b}_{T, j, a}$ for $j=1, j=10$ and $a=1$

These simulations precisely match the results obtained in the theoretical part of the paper. 


\section{REFERENCES}

[1] G. Da Prato, J. Zabczyk, Stochastic Equations in Infinite Dimensions, Cambridge University Press, Cambridge, 1992.

[2] M. Huebner, B. L. Rozovskii, On asymptotic properties of maximum likelihood estimator for parabolic stochastic PDE's, Probability Theory and Related Fields 103 (1995), no. 2, $143-163$.

[3] S. M. Iacus, Simulation and Inference for Stochastic Differential Equations, Springer Series in Statistics, 2008.

[4] J. Janák, Parameter estimation for stochastic partial differential equations of second order, Applied Mathematics and Optimization - submitted to, https:// arxiv.org/abs/1806.04045.

[5] T. Koski, W. Loges, On identification for distributed parameter systems, Stochastic Processes - Mathematics and Physics II, Proceedings of the 2nd BiBoS Symposium (1985), 152-159.

[6] T. Koski, W. Loges, Asymptotic statistical inference for a stochastic heat flow problem, Statistics \& Probability Letters 3 (1985), no. 4, 185-189.

[7] Y. A. Kutoyants, Statistical Inference for Ergodic Diffusion Processes, Springer, London, 2004.

[8] W. Liu, S. V. Lototsky, Estimating speed and damping in the stochastic wave equation, https://arxiv.org/abs/0810.0046

[9] W. Liu, S. V. Lototsky, Parameter estimation in diagonalizable stochastic hyperbolic equations, https://arxiv.org/abs/0906.4353

[10] B. Maslowski, J. Pospíšil, Ergodicity and parameter estimates for infinite-dimensional fractional Ornstein-Uhlenbeck process, Applied Mathematics and Optimization 57 (2008), no. 3, 401-429.

[11] B. Maslowski, C. A. Tudor, Drift parameter estimation for infinite-dimensional fractional Ornstein-Uhlenbeck process, Bulletin des Sciences Mathématiques 137 (2013), no. 7, 880-901.

[12] C. A. Tudor, F. G. Viens, Statistical aspects of the fractional stochastic calculus, The Annals of Statistics 35 (2007), no. 3, 1183-1212.

Department of Mathematics, University of Economics in Prague, Ekonomická 957, 14800 Prague 4, Czech Republid

E-mail address: janj04@vse.cz 